RECENT RESULTS ON INCLUSIVE REACTIONS ${ }^{*}$

F. T. Dao ${ }^{\dagger}$

Fermi National Accelerator Laboratory

Batavia, Illinois 60510

September 1974

* Review talk given at the 1974 Meeting of the Division of Particles and Fields of the American Physical Society, Williamsburg, Virginia. †Address after October 1974: Physics Department, Tufts University, Medford, Massachusetts 02155. 


\title{
RECENT RESULTS ON INCLUSIVE REACTIONS
}

\author{
F. T. Dao \\ Fermi National Accelerator Laboratory \\ Batavia, Illinois 60510
}

\begin{abstract}
Data concerning charged particle multiplicities, correlations between particles and single-particle inclusive distributions produced in high-energy collisions is reviewed.
\end{abstract}

\section{INTRODUCTION}

With accelerators at Fermilab, Serpukhov, and ISR all working, we have accumulated a tremendous amount of data on inclusive reactions-reactions in which one or at most a few particles are studied at one time. We should not forget the story of three blind men who are given an elephant and asked to describe the animal. All come up with different descriptions. In inclusive studies, we are like the blind men.

Fortunately with the ingenuity of physicists, we are catching and at the same time identifying more and more of the particles in a given reaction. We are indeed approaching the era of semi-inclusive reactions-reactions in which we know the general form of the reaction via the knowledge of the number of charged particles $n_{c}$ involved.

For this review I have selected the following three topics for discussion, partly due to my limited knowledge and partly due to the possibility that each topic may give us insight into the production mechanism in high-energy collisions:

I. GROSS FEATURES - MULTIPLICITY DISTRIBUTIONS

II. CORRELATION STUDY

III. SINGLE-PARTICLE INCLUSIVE SPECTRA 


\section{GROSS FEATURES - MULTIPLICITY DISTRIBUTIONS}

(a) Average Value

The multiplicity distribution of charged particles has been accurately determine from experiments performed at Fermilab, Serpukhov, and ISR. ${ }^{1}$ It is noted that (i) the average value $\left\langle\mathrm{n}_{\mathrm{c}}\right\rangle$ in the distribution increases slowly with the laboratory momentum, and (ii) the shape of the distribution, once the dependence of the average value is taken out, does not appear to change with the incident energy and the initial state.

To examine the first point closely, we study $\left\langle\mathrm{n}_{\mathrm{c}}\right\rangle$ as a function of laboratory momentum. Figure 1 shows the average value in the range $10 \mathrm{GeV} / \mathrm{c}$ to $10 \mathrm{TeV} / \mathrm{c}$ for all available data. Also included in the figure are estimates from ISR and a recent estimate from a cosmic ray experiment. The figure shows that $\left\langle n_{c}\right\rangle$ varies very slowly with $p_{1 a b}$. The four recent measurements at Fermilab $(100,200,300$, and $400 \mathrm{GeV} / \mathrm{c}$ pp) together with the Serpukhov data $(50,69 \mathrm{GeV} / \mathrm{c})$ can be fitted with a in $\mathrm{p}_{1 \mathrm{ab}}$ dependence. However for all the data above $12 \mathrm{GeV} / \mathrm{c}$, the increase in $\left\langle\mathrm{n}_{\mathrm{c}}\right\rangle$ is faster than $\mathrm{tn} \mathrm{p}_{1 a b}$. Two curves are given in the figure and they represent fits to the pp data only.

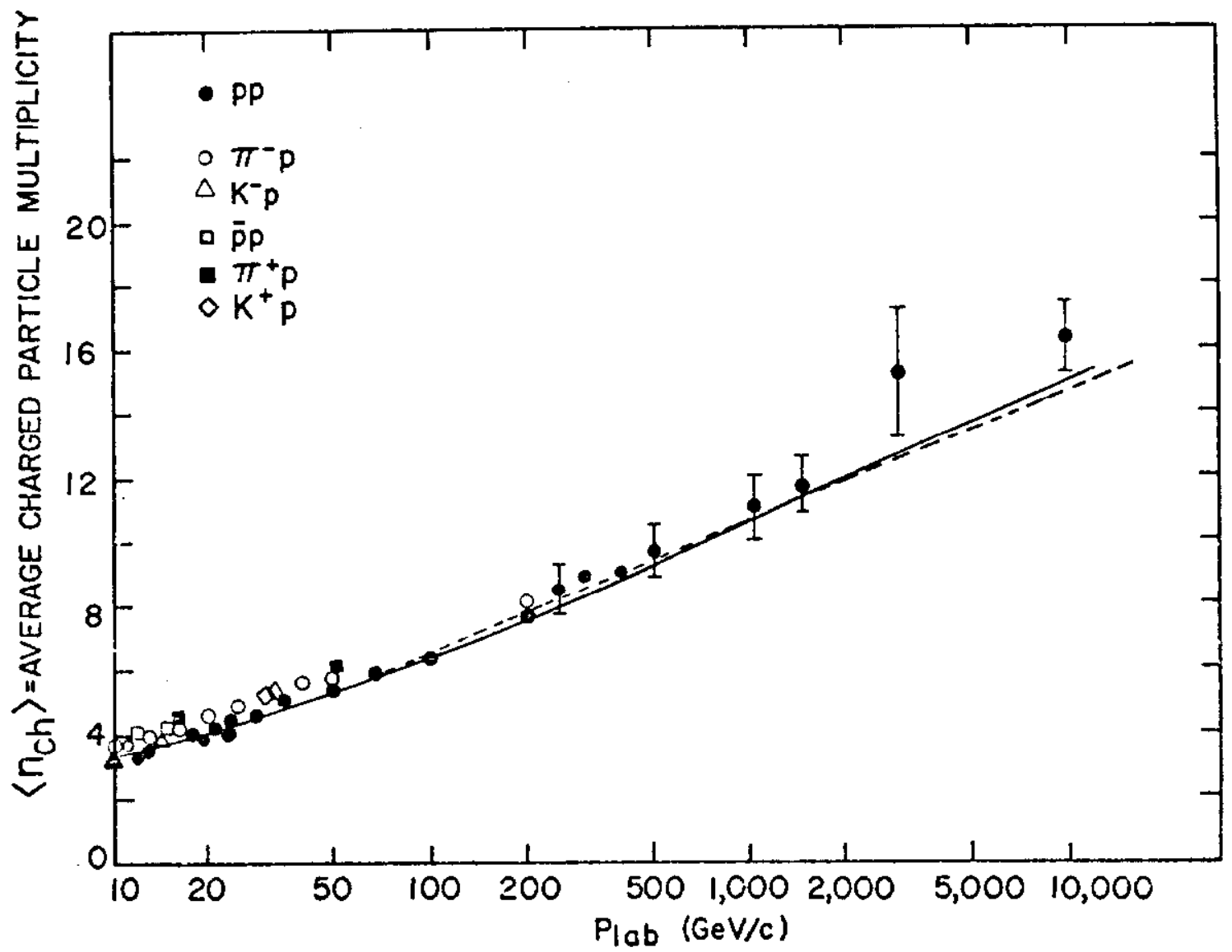

Fig. 1 
The figure also shows very weak dependence of $\left\langle n_{c}\right\rangle$ on the initial state. This is especially true if the low-energy data are plotted as a function of the $\mathrm{cm}$ energy squared $\mathrm{S}$, as given in Fig. 2. ${ }^{2}$ A great variety of initial states are represented, and one might reasonably expect individual differences. However, the value of the average multiplicity and its S-dependence are remarkably similar. This suggests that in high-energy collisions, the excited state is quickly thermalized and the final state multiplicity depends only on the center-of-mass energy.

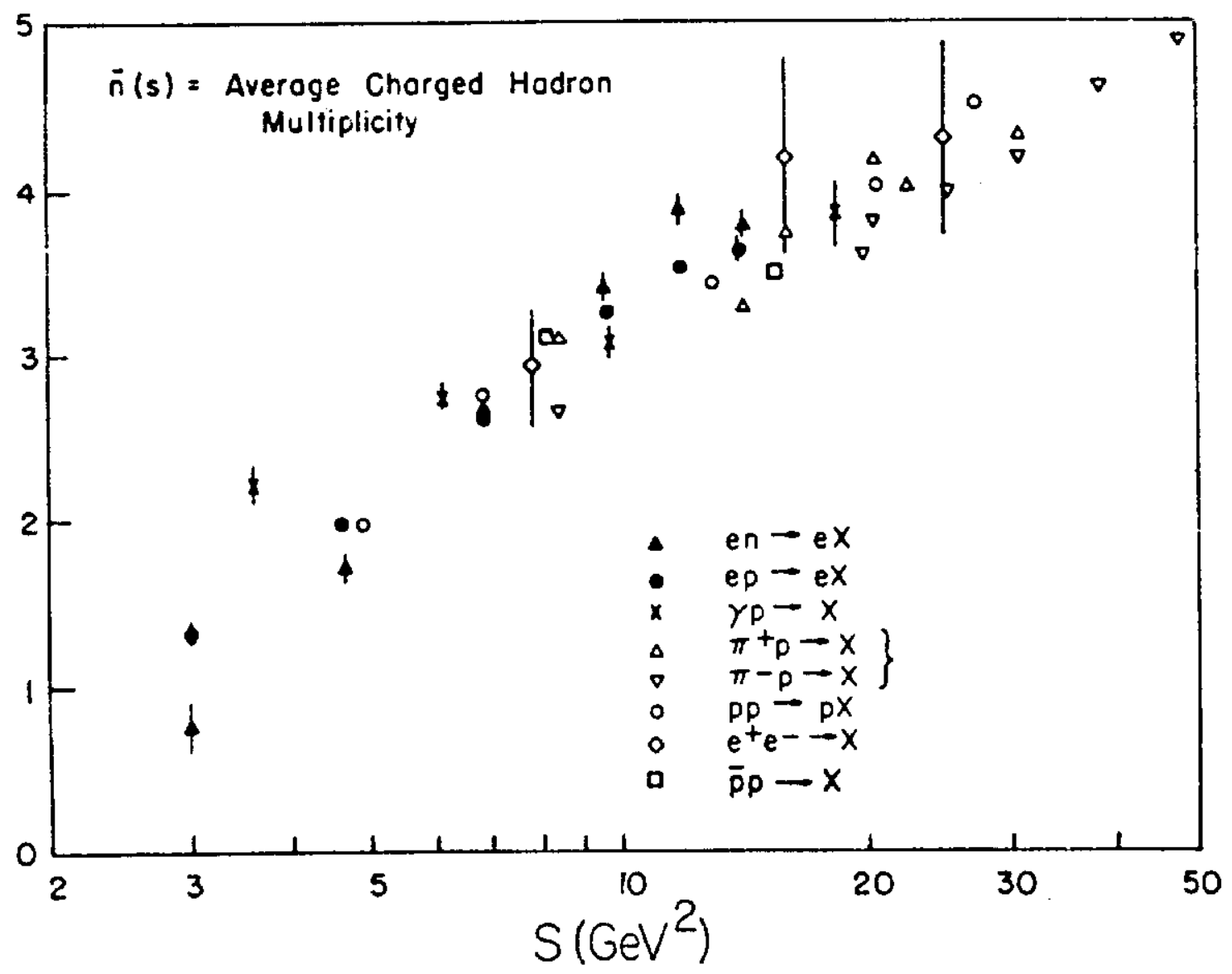

Fig. 2

(b) Shape Independence

The shape of the multiplicity distribution was first predicted by Koba-Nielsen-Olesen $(\mathrm{KNO})^{3}$ to be independent of energy $(\sqrt{\mathrm{s}})$ at sufficiently high energy,

$$
\frac{1}{\sigma} \frac{d \sigma}{d\left(\frac{n}{\langle n\rangle}\right)} \underset{s \rightarrow \infty}{\longrightarrow} \psi\left(\frac{n}{\langle n\rangle}\right)
$$


where the function $\psi$ does not have explicit dependence on $\sqrt{\mathrm{s}}$. Slattery ${ }^{4}$ first showed in 1972 that the pp data in the 50-300 GeV/c range supported the KNO scaling. Subsequently many papers have been written on this subject in an attempt to prove, disprove, or improve the KNO scaling. An excellent review has been given by Wroblewski ${ }^{5}$ at the Zakopane Summer School in 1973. He made a very nice observation that Eq. (1) scales much better if $n-1 /<n-1\rangle$ is used instead of $n /<n>$.

Figure 3 shows the high energy multiplicity data plotted in the $n /<n\rangle$ variable. The figure shows that $\pi p$ and $p p$ interactions look alike as far as the multiplicity distributions are concerned even though their inelastic cross sections are different. The solid curve derives from a local excitation model of Koba and Buras ${ }^{6}$ which predicts:

$$
\psi(z)=\pi z e^{-\frac{\pi}{4} z^{2}}
$$

where $\mathrm{z}=\mathrm{n} /<\mathrm{n}\rangle$. This model assumes that the hadron-hadron collision consists of collisions between a number of constituents of one hadron and more of the other.

Figure 4 shows that this kind of scaling concerning the shape of the distribution is not unique to particle physics. Back in 1957 Wigner speculated that the distribution of the level spacings in complex nuclei when scaled by the mean value follows the function given in Eq. (2). Subsequently it was found experimentally that this Wigner scaling also holds in complex atomic spectra. ${ }^{8}$ (The Wigner scaling in atomic and nuclei spectra has been shown to arise from general statistical argument rather than the specific form of interaction.)

\section{CORRELATION STUDY}

There is much interest in studying how particles are correlated in multiparticle production. Over the past two years, tremendous amount of data has come from Fermilab, Serpukhov, ISR, and other laboratories. 1 Emphasis is usually made on the two-particle correlation. In this review, I shall go over some of the se data and examine the essential question: Is there evidence for correlation in the highenergy data? If there is, how strong is it? And what are the sources of this correlation? 

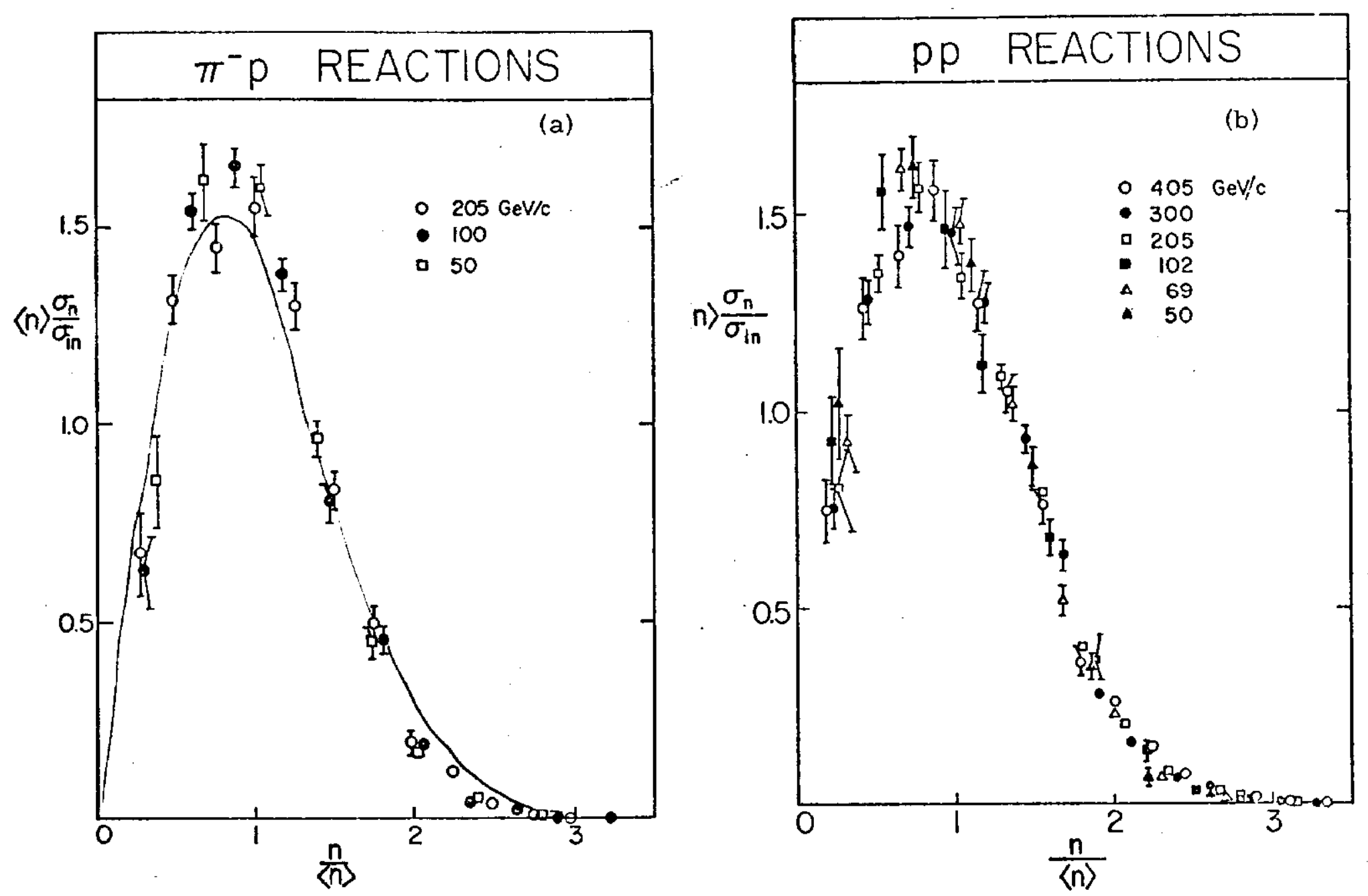

Fig. 3 

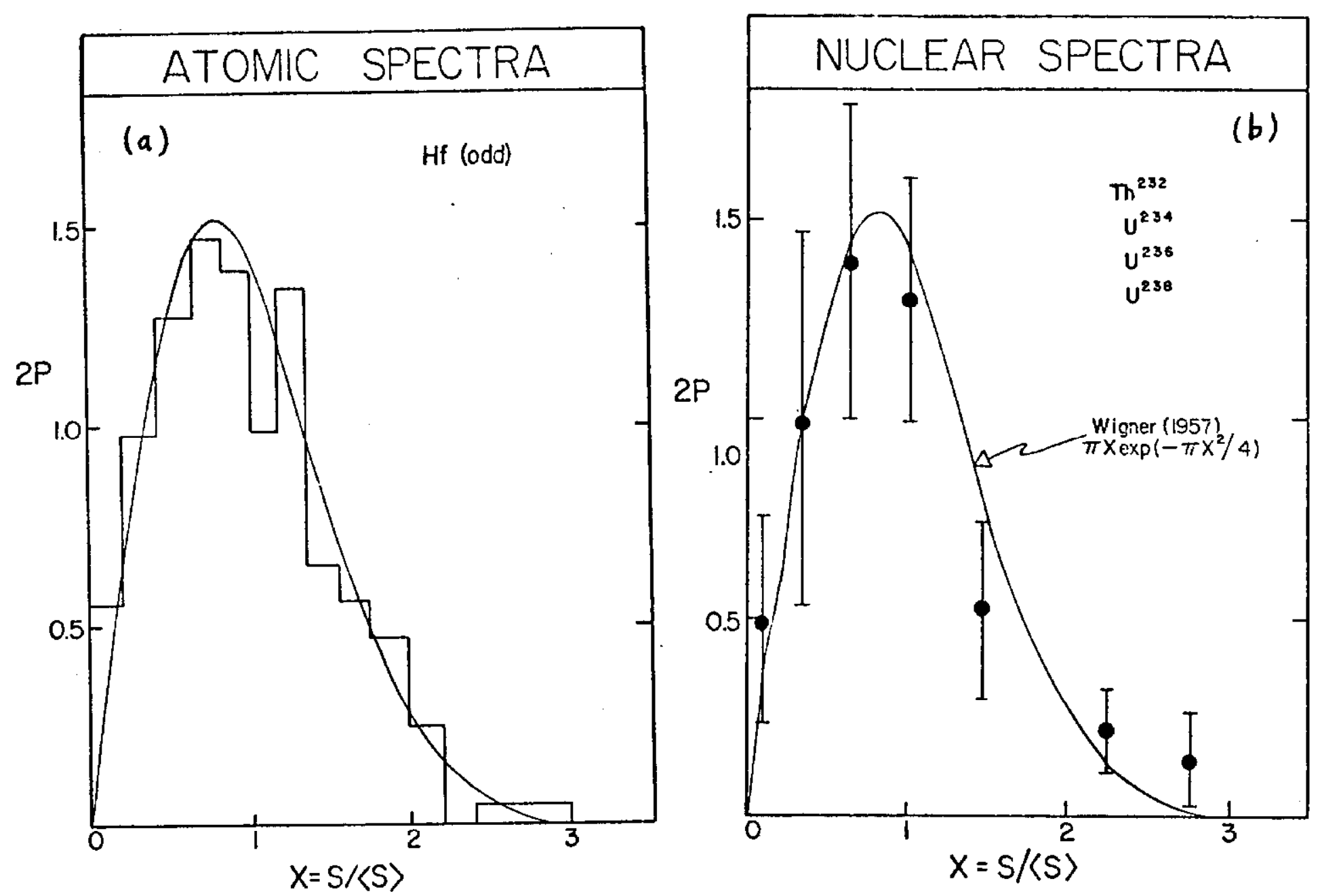

Fig. 4 
(a) Resonance Production

The simplest but not necessarily the easiest kind of correlation to search for in high-energy collisions is resonance production.

Winkelman ${ }^{9}$ has presented data at the recent Meson Spectroscopy Conference on the $\rho^{0}$ production in $p p$ and $\pi p$ interactions from $\sim 10$ to 200 $\mathrm{GeV} / \mathrm{c}$. Figure 5 shows the invariant mass distribution of $\pi^{+} \pi^{-} \mathrm{com}-$ bination from $205 \mathrm{GeV} / \mathrm{c} \pi^{-} \mathrm{p}$ and $205 \mathrm{GeV} / \mathrm{c}$ pp interactions. An enhancement is observed at the $p$ mass region for both interactions. The average number of $p^{\circ}$ produced per inelastic $\pi p$ and pp collisions is estimated at 0.5 and $0.2^{10}$ respectively. This number should be compared with an average of eight charged particles, most of which are pions. Because of the combinational problems, search for resonances which decay into more than two charged pions are prohibitive. It appears that the majority of the pions do not come from resonances.

(b) Production Correlation

The associated production of $\pi^{\circ}$ is observed to increase linearly with the prong multiplicity at high energies. As most of the charged tracks are pions, these data tend to show that neutral and charged pions are highly correlated in production. Figure 6 shows some recent data ${ }^{11}$ of $\left\langle\mathrm{n}_{\mathrm{o}}\right\rangle$ plotted against $\mathrm{n}$ (which as a first approximation is $\mathrm{n}_{-}$). Figur 7 shows an earlier ${ }^{\top}$ compilation taken from Ref. 1. The "Slopes $\alpha$ are results of linear fits to the data using the form $\left\langle\mathrm{n}_{\pi_{0}}\right\rangle=\alpha \mathrm{n}_{-}+\beta$. The $\alpha^{\prime}$ s increase with the incident energy. Note that the $\mathrm{e}^{\pi} \alpha$ for the 15 $\mathrm{GeV} / \mathrm{c}$ pp data are much bigger than the corresponding value for the 12 or $19 \mathrm{GeV} / \mathrm{c}$ pp data. Many theoretical models have been constructed to "explain" these data and the energy dependence. ${ }^{12}$ They usually involve some form of "clusters" as input. However, the se clusters are very elusive and difficult to determine experimentally.

Figure 8 shows $\left\langle\mathrm{n}_{\mathrm{K}_{\mathrm{s}}}>\right.$ and $\left\langle\mathrm{n}_{\mathrm{N}^{\mathrm{O}}}>\right.$ as a function of $\mathrm{n}_{-}$. They show the same behavior; namely the slope increases with energy. Note that the slope in $\mathrm{K}_{s}^{0}$ production is expected to be smaller by a factor of $\sigma\left(\mathrm{K}_{\mathrm{S}}^{\mathrm{O}}\right) / \sigma\left(\pi^{\mathrm{O}}\right)$ than the corresponding slope of $\pi^{\mathrm{O}}$ at the same energy.

This correlation in associated production has also been studied in terms of the Muller moment $f_{2}^{\mathrm{AB}}$ :

$$
f_{2}^{A B}=\left\{\begin{array}{l}
<n_{A} n_{B}>-<n_{A}><n_{B}>\text { if } A \neq B \\
<n_{A}\left(n_{A}-1\right)>-<n_{A}>\text { if } A=B .
\end{array}\right.
$$


(a)

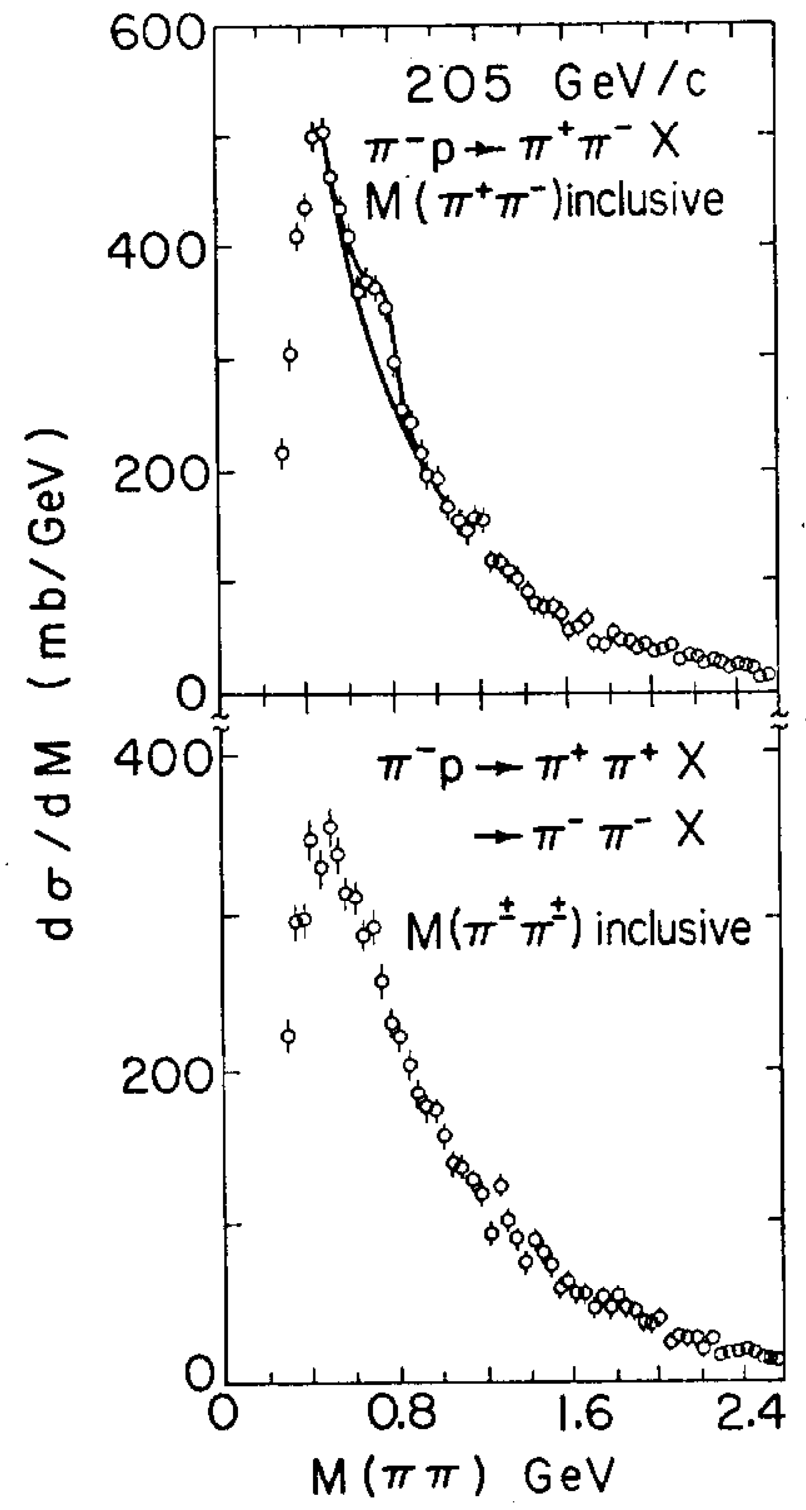

(b)

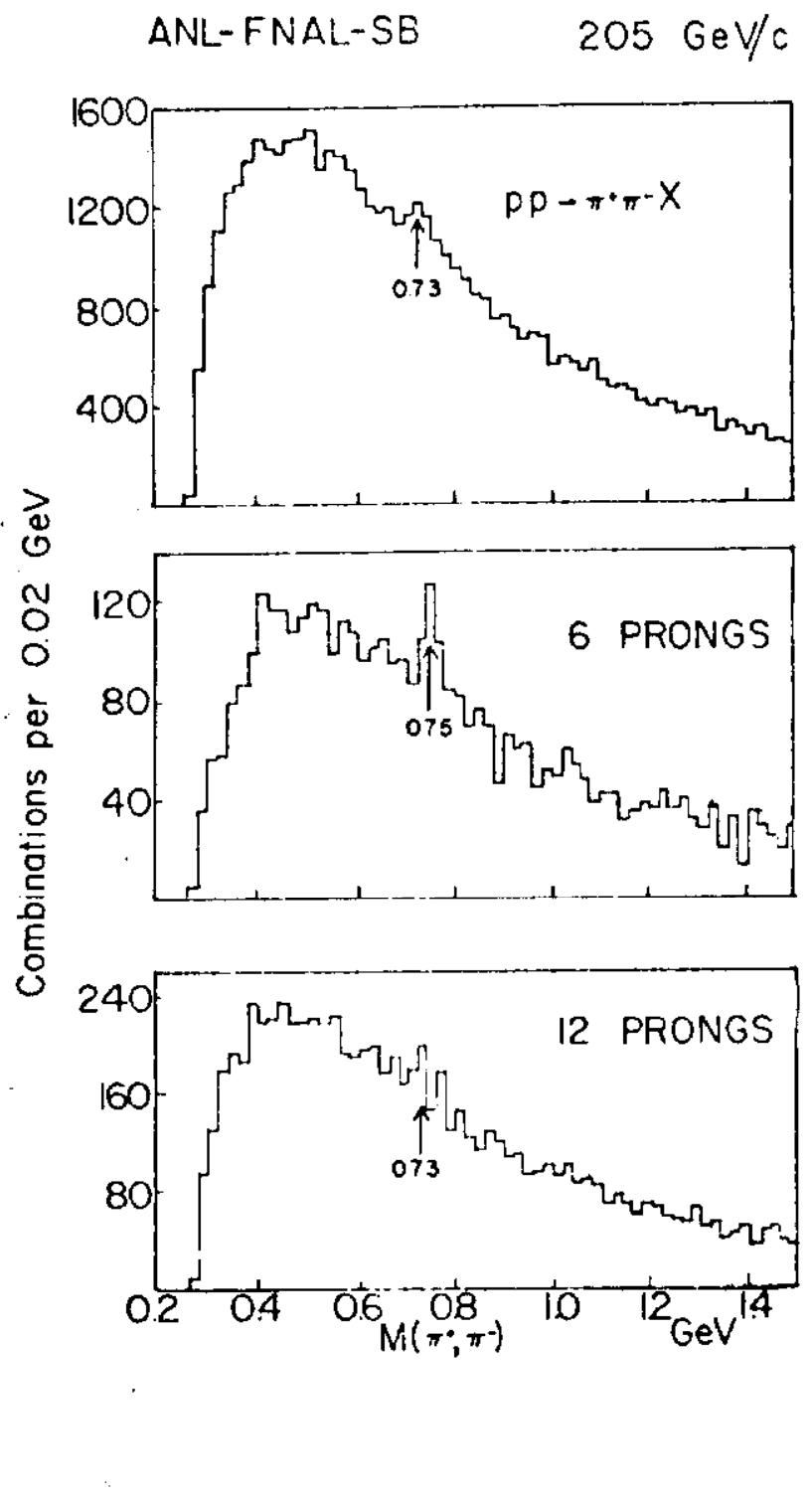

Fig. 5 


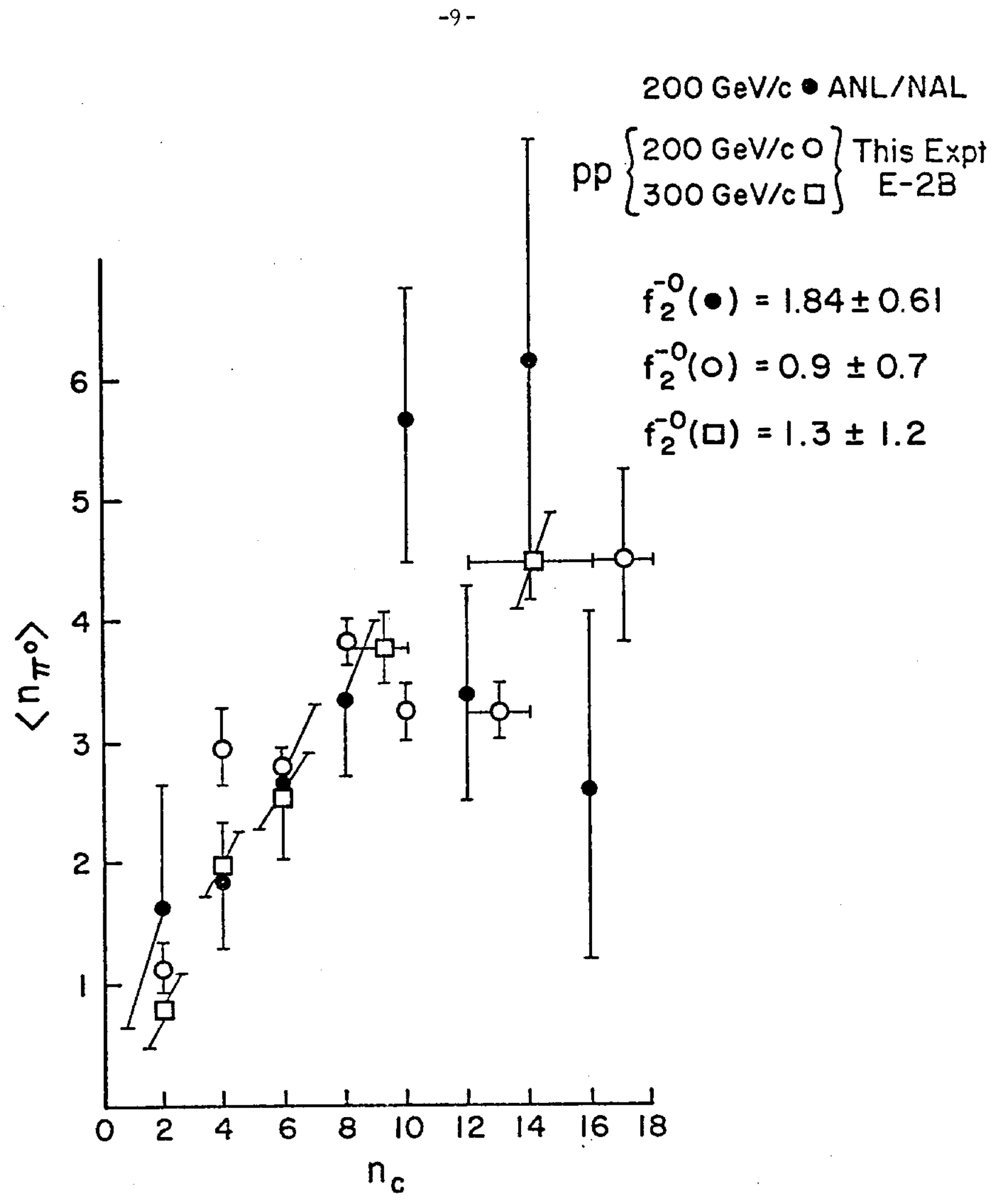

Fig. 6 

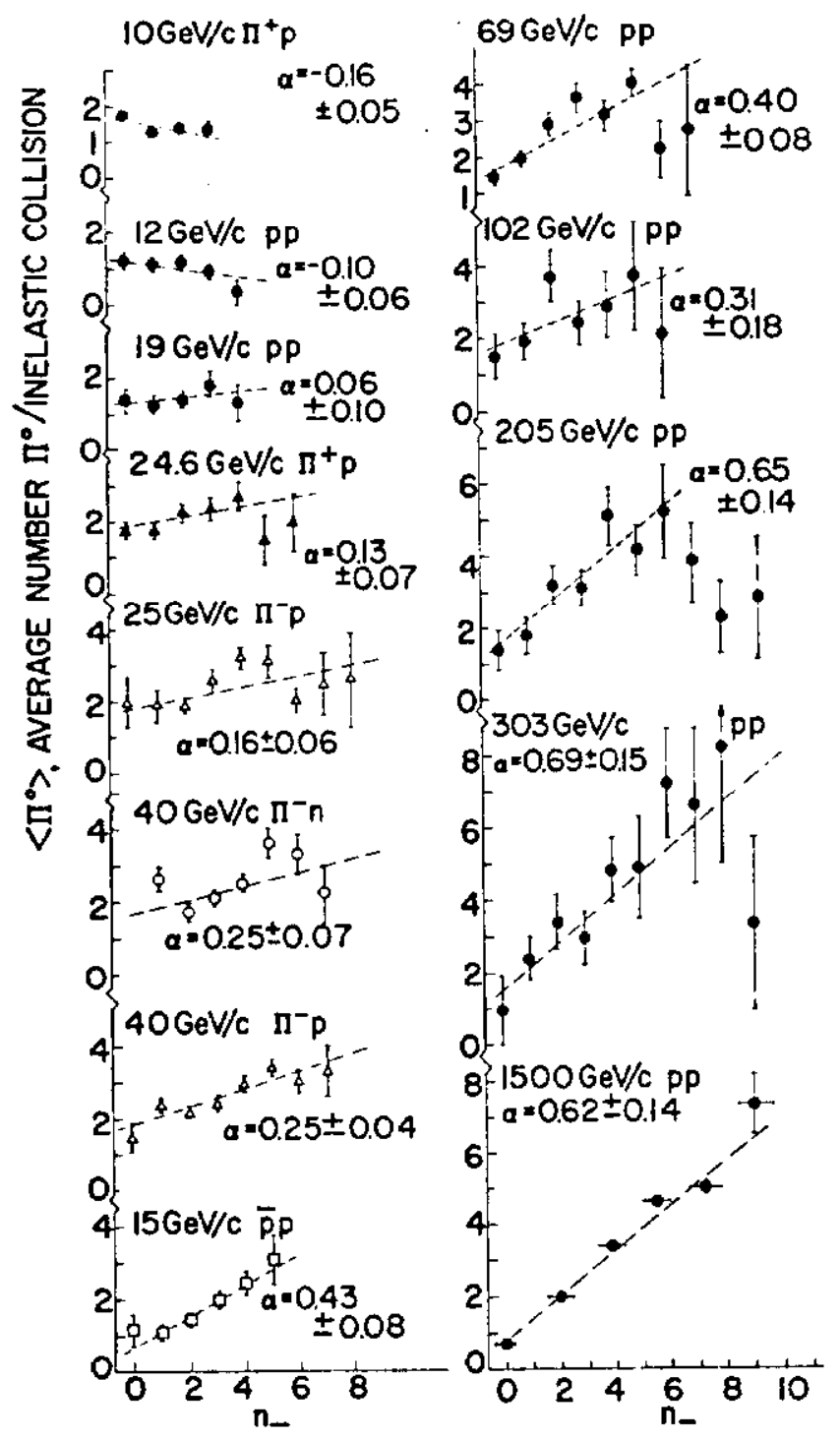

$205 \mathrm{GeV} / \mathrm{c} \mathrm{pp}$

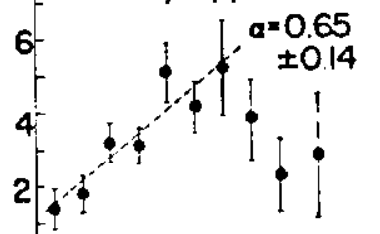

Fig. 7
Note that $f_{2}{ }^{\mathrm{AB}}=0$ if there is no correlation. Figure 9 shows $f_{2}^{\Lambda^{8} \pi^{-}}$and $f \Lambda^{\Lambda^{\circ}} \pi^{-}$as a function of the laboratory momentum. The data again indicates an increasing correlation with the incident energy.

Recently a study has been made on the associated $\pi^{\circ}$ production from the $15 \mathrm{GeV} / \mathrm{c} \overline{\mathrm{p}} \mathrm{p}$ interactions in an effort to understand the cause of this strong correlation between $\pi^{\circ}$ and $\pi^{-} \cdot 13$ Figure 10 shows $\langle\mathrm{n}$ o $>$ versus $\mathrm{n}_{\text {- from the inclusive data and }}$ the data which have been separated into annihilation and nonannihilation components by ionization. The figure shows that $\left.\left.\left\langle\mathrm{n}_{\mathrm{o}}\right\rangle\right\rangle_{\text {nn }}\right\rangle\left\langle\mathrm{n}_{\mathrm{o}}\right\rangle_{\text {non }}$ but each component does not show much dependence on $n_{-}$. This study shows an important fact that the dynamics of correlation in high-energy collision can be induced by the presence of more than one component in an interaction even though each component may not have strong correlation in its own. Likewise in high energy hadronhadron collisions, the presence of diffractive and nondiffractive components may be largely responsible for the positive correlation.

\section{(c) Rapidity Correlation}

Extensive study has been made on the two-particle correlation in the rapidity space. ${ }^{15}$ Data from Fermilab, Serpukhov, and ISR tend to establish a pattern generally in support of the short-range correlation as predicted in the Muller-Regge theory. It should be emphasized that the se data (and hence evidence) have been mostly examined in the inclusive reactions. 


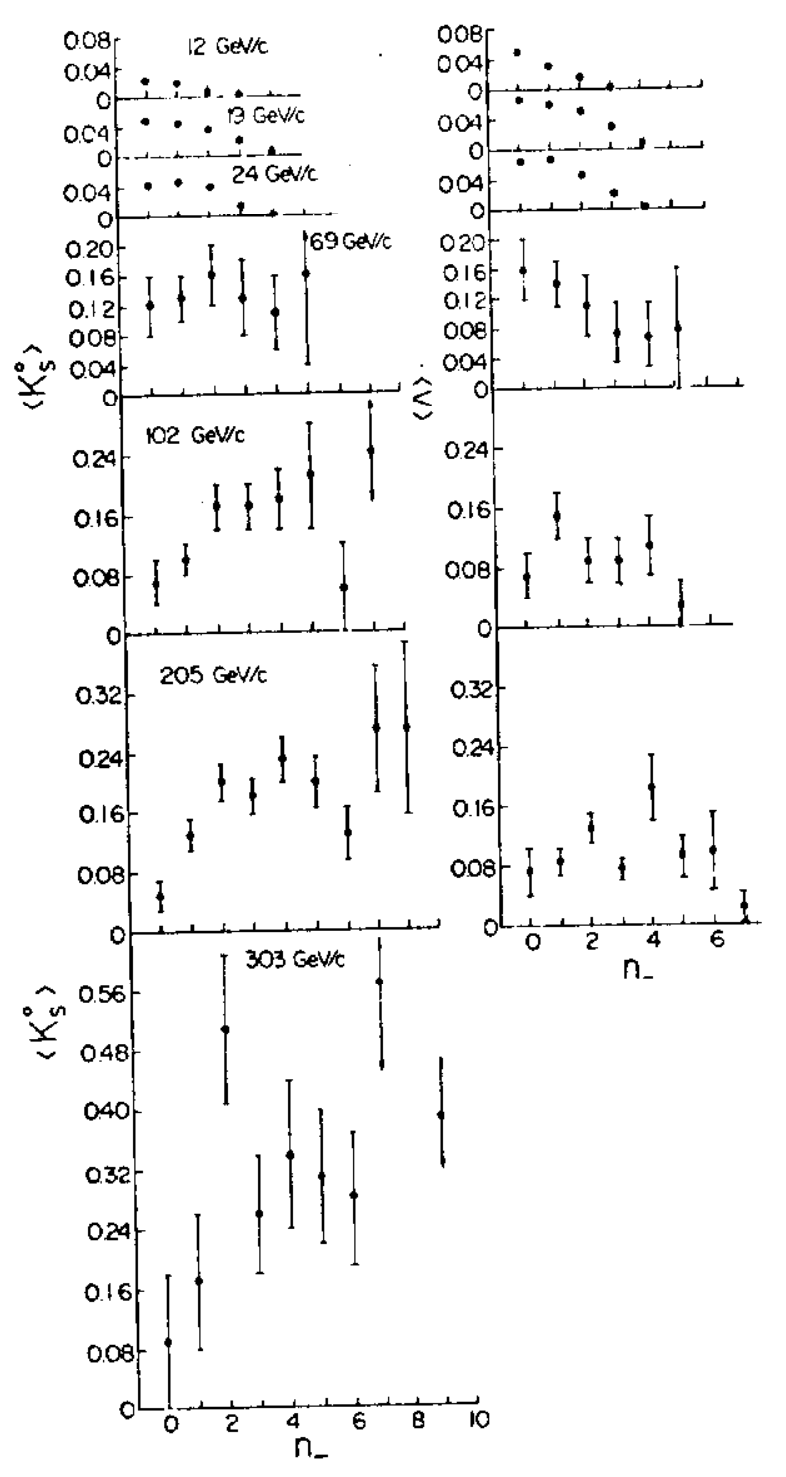

Fig. 8
The correlation functions $\mathrm{R}$ and $\mathrm{C}$ are generally used. They are defined as

$R\left(y_{1}, y_{2}\right) \equiv \frac{\rho\left(y_{1}, y_{2}\right)}{\rho\left(y_{1}\right) \rho\left(y_{2}\right)}-1=\frac{C\left(y_{1}, y_{2}\right)}{\rho\left(y_{1}\right) \rho\left(y_{2}\right)}$

where $\rho\left(\mathrm{y}_{1}, \mathrm{y}_{2}\right)=\sigma_{\mathrm{in}}^{-1} \mathrm{~d} \sigma / \mathrm{dy}_{1} \mathrm{dy} \mathrm{y}_{2}$ and $\rho\left(y_{1}\right)=\sigma_{i n}-1 \mathrm{~d} \sigma / \mathrm{dy}_{\mathrm{i}}$.

Figure 11 shows a contour plot of $R_{12}$ and its dependence on $\mathrm{y}_{1}$ and $\mathrm{y}_{2}$ for all charged combinations from the $205 \mathrm{GeV} / \mathrm{c}$ pp interactions. 16 Similar plots have been made at other energies. They are generally taken as giving strong support for the Muller-Regge description of a positive short-range correlation in the central region.

Figure 12 shows the correlation function for various charged states as a function of the rapidity difference. These data show a rapid fall-off when the difference in $\left|y_{1}-y_{2}\right|$ becomes large. This behavior however holds for negative-negative and positivepositive pairs as well as positivenegative pairs. In the simple Muller-Regge picture the last two charge combinations are not expected to have short-range $\overrightarrow{\text { correlations. }}$

Recent results 16,17 on semi-inclusive rapidity correlation, moreover, cast doubt on the current strong short-range correlation picture. In a semi-inclusive reaction, the number of charged particles $\left(n_{c}\right)$ is also recorded. In other words, the correlation function $R$ is computed for each sample of events with the same $n_{c}$. This additional information is crucial in two respects: (1) for $n_{c}$ small, the correlation probably arises from diffractive processes and for large $n_{c}$, short-range correlation is expected to predominate; (2) events with the same $n_{c}$ have similar energy-momentum constraint. 


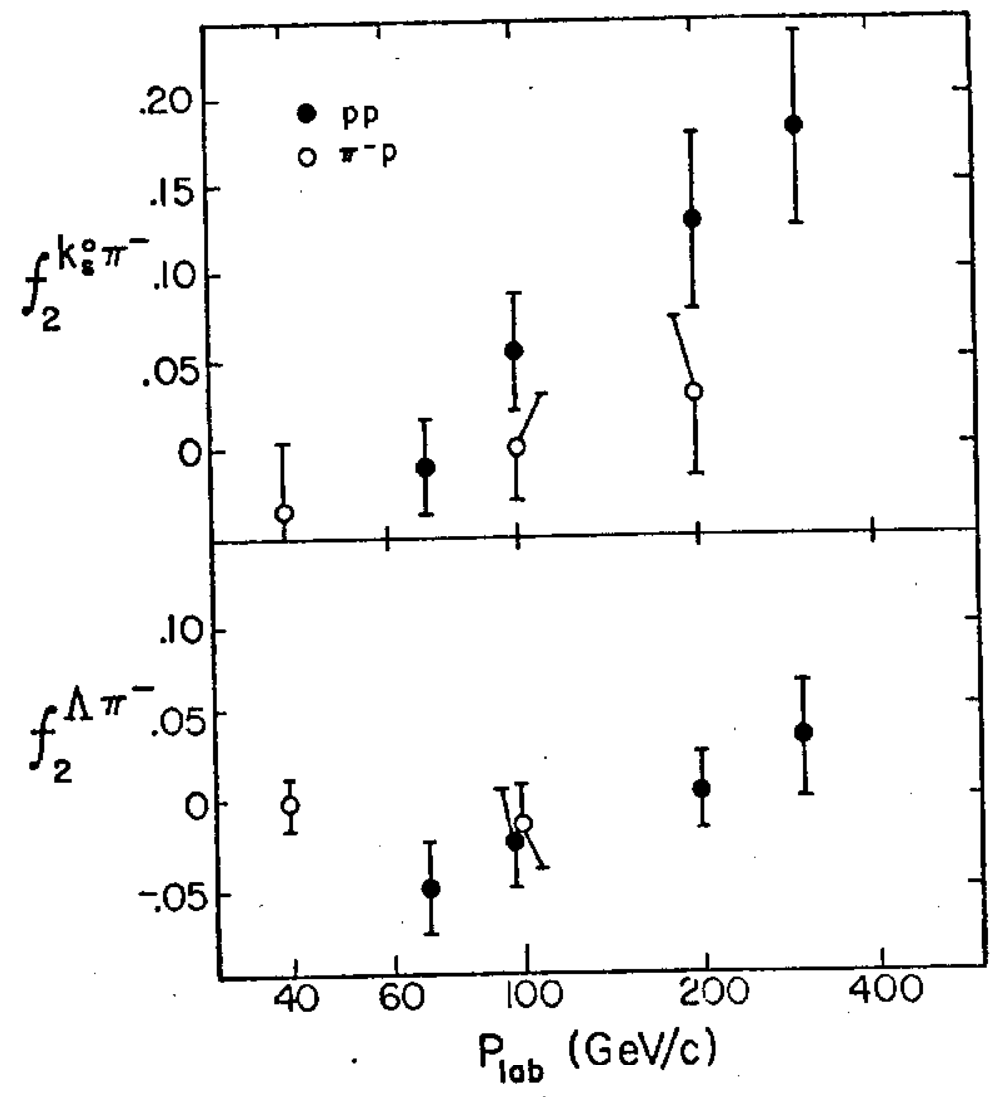

Fig. 10

Fig. 9

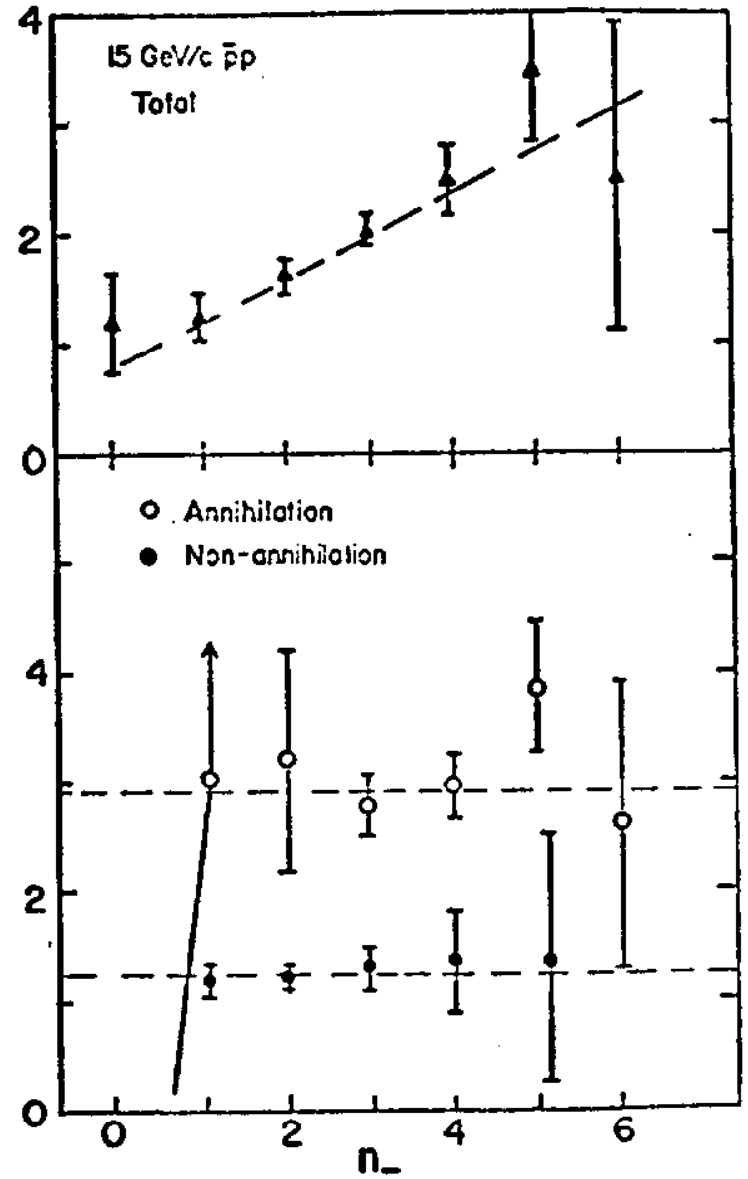


$R_{c c}$ FOR $205 \mathrm{GeV} / \mathrm{c}$ PP

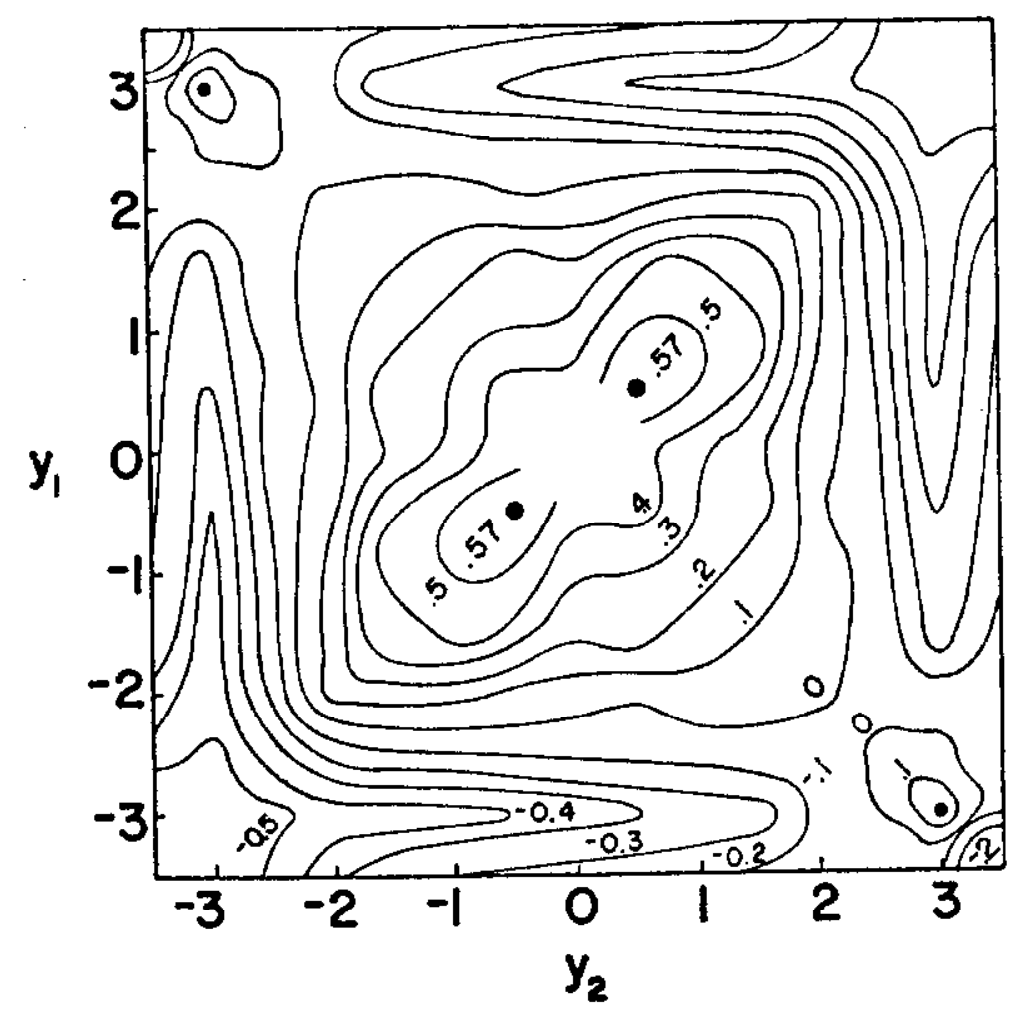

Fig. 11

Figures 13 and 14 show the semi-inclusive distributions for $\pi^{-} \pi^{-}$ and $\pi^{+} \pi^{-}$pairs. In contrast to the (total) inclusive distribution, none of these distributions show any strong short-range correlations. With the notable exception of 4-pronged events with $\pi^{+} \pi^{-}$pairs, they are consistent with little or no correlation. If the short-range two-particle correlation as predicted by the Muller-Regge theory is a genuine effect, it should be present regardless of the number of charged particles in the final state, just as $\rho$ resonance is observed in 4-pronged as well as 6pronged events. Similar results have been observed in the correlation between $\gamma$ and charged particles. Figure 15 shows the preliminary data of the inclusive and semi-inclusive distributions of $R_{\gamma c}$ and $R_{c c}$ from the wide-gap spark chamber collaboration. 1

It is likely that the structure observed in the inclusive distribution for incident momentum below $400 \mathrm{GeV} / \mathrm{c}$ is mainly due to the kinematics. To check this point, Singer et al. ${ }^{18}$ have done a Monte Carlo calculation using the single-particle rapidity and transverse momentum distribution for each topology and imposing the energy-momentum conservation. They have been able to reproduce the general features of the observed twoparticle rapidity correlation in inclusive reaction at $205 \mathrm{GeV} / \mathrm{c}$. Their Monte Carlo result is shown in Fig. 16. 

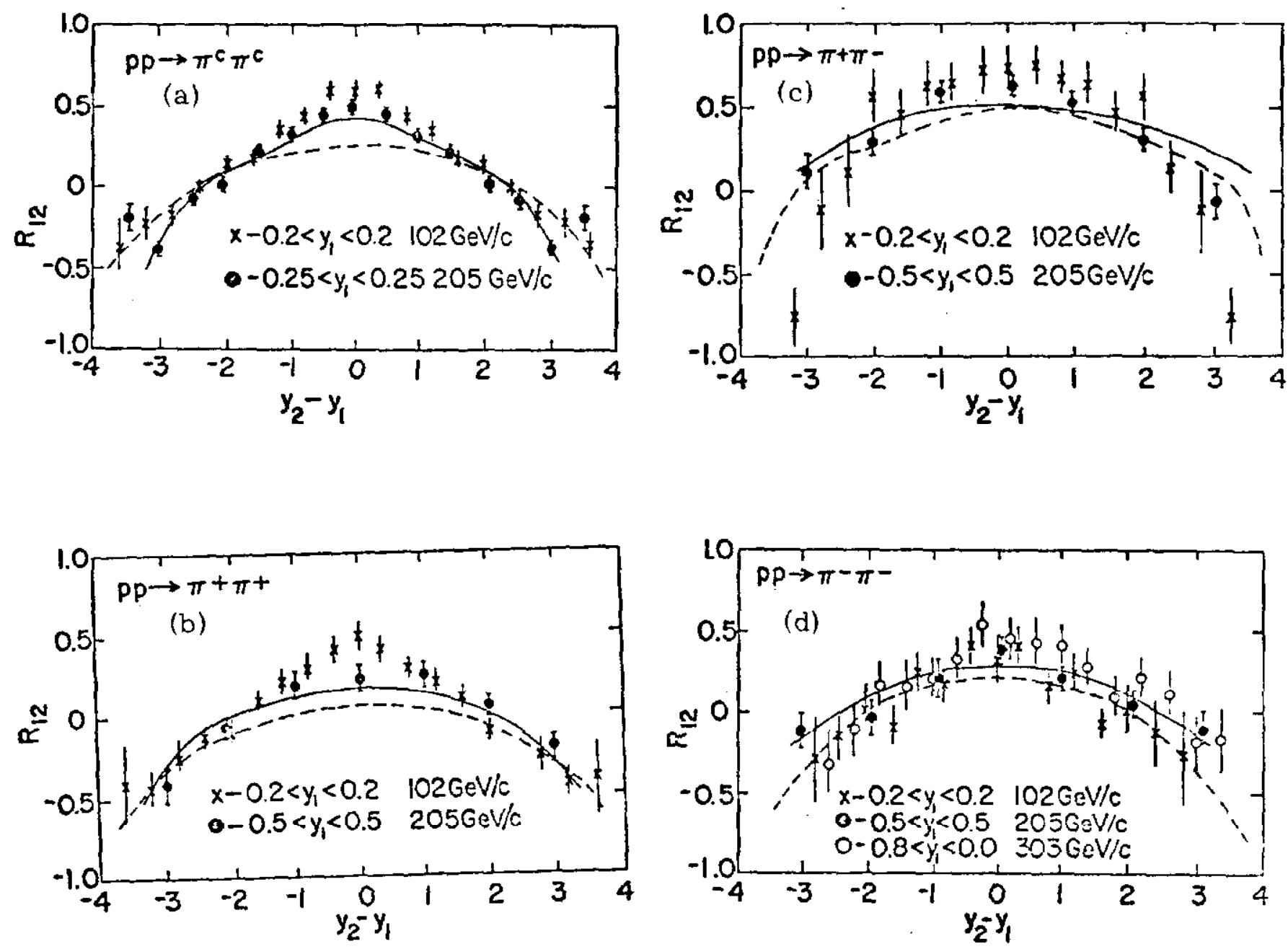

Fig. 12 
- $69 \mathrm{GeV} / \mathrm{c}-0.2<y_{1}^{-}<0.2$

- $205 \mathrm{GeV} / \mathrm{c}-0.5<\mathrm{y}_{1}^{-}<0.5$

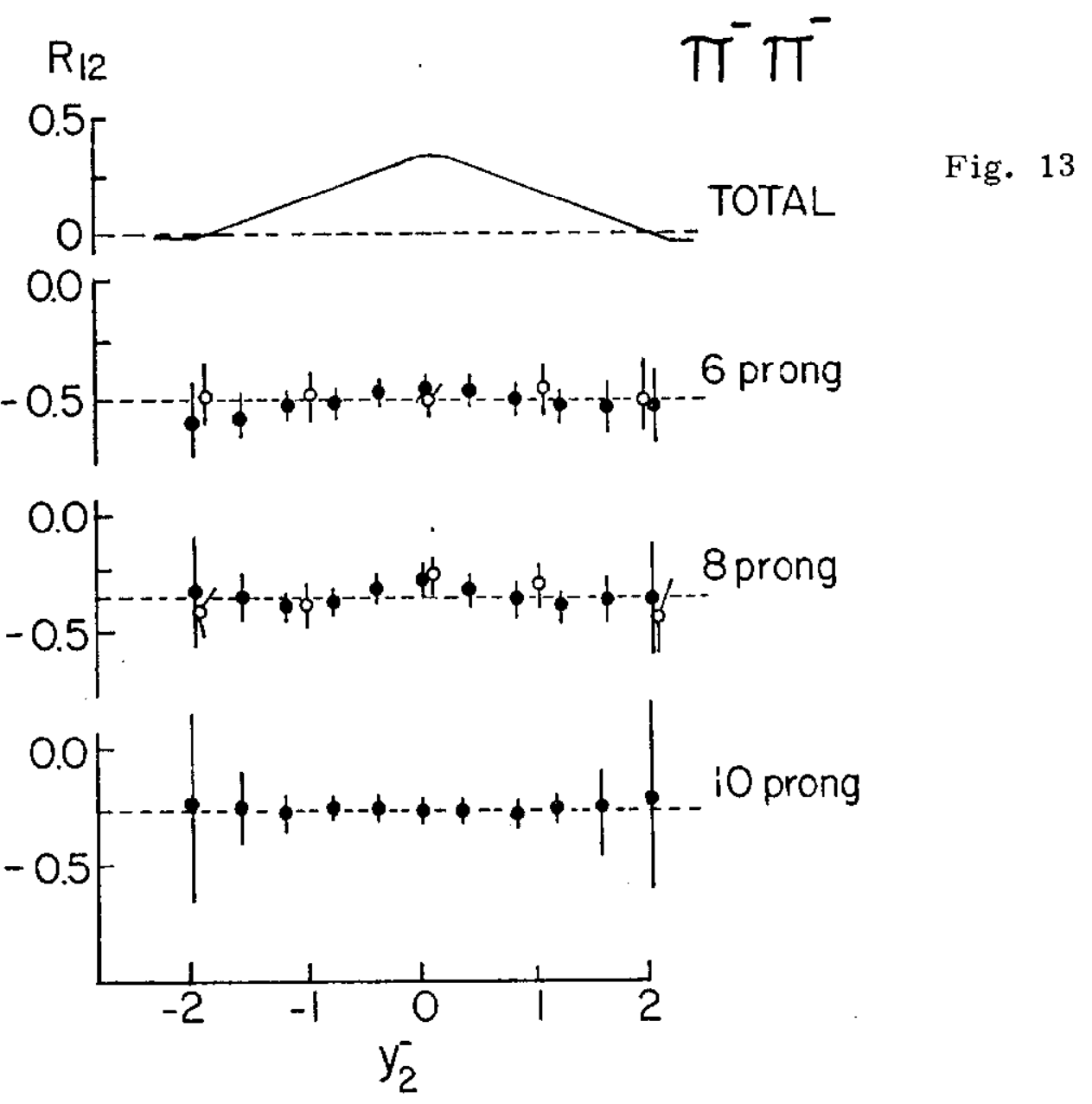

Another surprising result from the recent semi-inclusive studies give evidence that the low-multiplicity events rather than the high multiplicity ones tend to have correlation in the central region. Figure 17 shows the value of the semi-inclusive correlation at $y_{1}=y_{2}=0 R_{n}\left(y_{1}, y_{2}\right)$ as a function of $p_{1 a b} \cdot 17,18$ Whereas $R_{n}(0,0) \simeq 0$ for $n \geq 6, R_{4}(0,0)$ increases with the incident momentum. This latter behavior for events with small $\mathrm{n}$ may be consistent with the fragmentation models which predict $R(0,0) \propto \sqrt{s}$.

(d) Azimuthal Correlation

Another correlation which is related to the rapidity correlation is the correlation induced by the transverse angles. The CERN- 


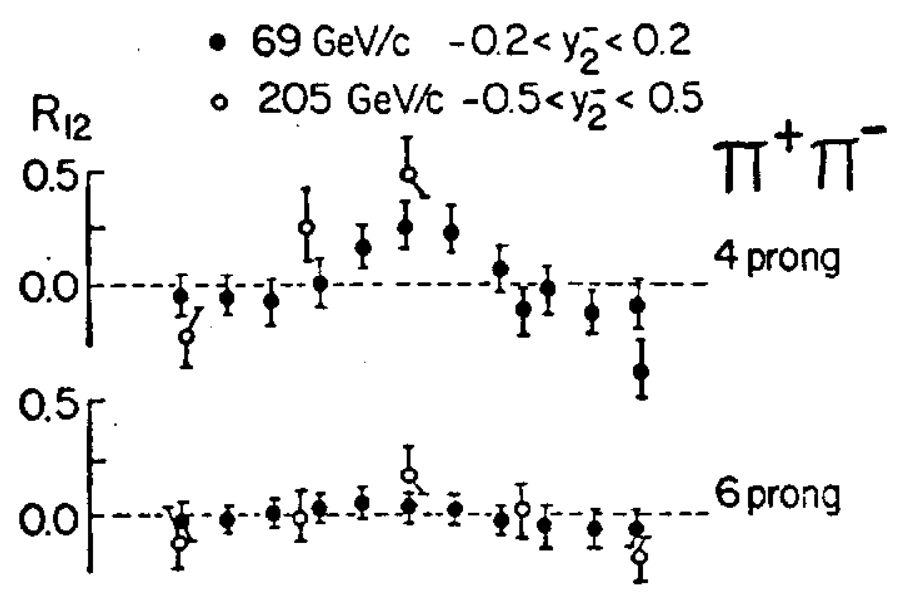

Hamburg -Vienna Collaboration $^{19}$ has measured the azimuthal correlations between photons and charged particles in order to establish whether the transverse momentum of a given particle is balanced in a limited region of $y$ around the selected particle (as required in the short-range correlation picture) or over the whole $y$-range (as re-

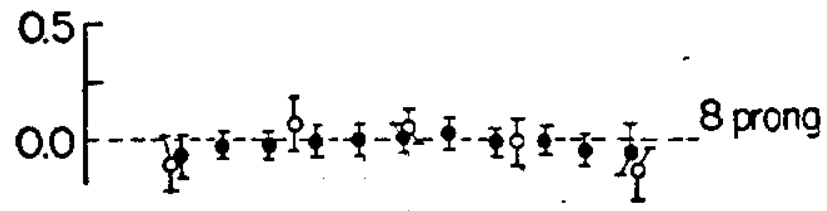
quired by the energy momentum conservation, as in the pionization or uncorrelated jet model). The azimuthal assymetry is expressed by

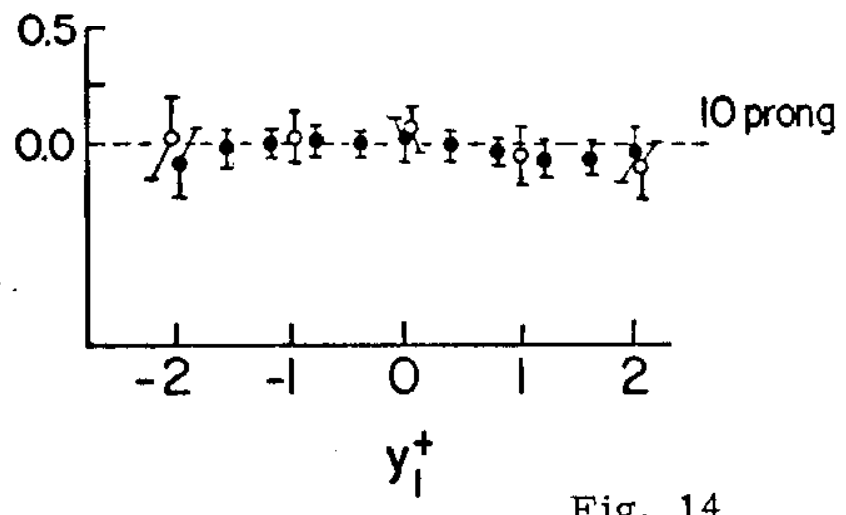
$F_{y c h}=a+b\left(p_{t}\right) \cos \phi$. Figure 24 shows that when the transverse momentum of the charged particle is changed, the momentum balance, as indicated by the factor $b$, is not affected only locally but in the whole $\mathrm{y}$ region. This indicates a long-range correlation effect.

The semi-inclusive azimuthal correlation has been studied by the Stony Brook-Argonne-Fermilab Collaboration. 20 The azimuthal angular distributions for individual topologies are shown in Fig. 19. It is observed that (a) the distributions are asymmetric and peak near $180^{\circ}$ and (b) the asymmetry becomes less pronounced as the charged multiplicity increases. It is interesting to note that the amount of asymmetry defined by

$$
A_{n}=\frac{\int_{\pi / 2}^{\pi} \frac{d \sigma_{n}}{d \phi} d \phi-\int_{0}^{\pi / 2 \frac{d}{n} \sigma_{n}} \frac{d \phi}{d \phi} d}{\int_{0}^{\pi \frac{d}{d} \sigma_{n}} \frac{d \phi}{d \phi}}
$$

agrees very well with the simple pionization model prediction of Friedman, Risk and Smith in their 1972 paper. ${ }^{21}$ Figure 20 shows 


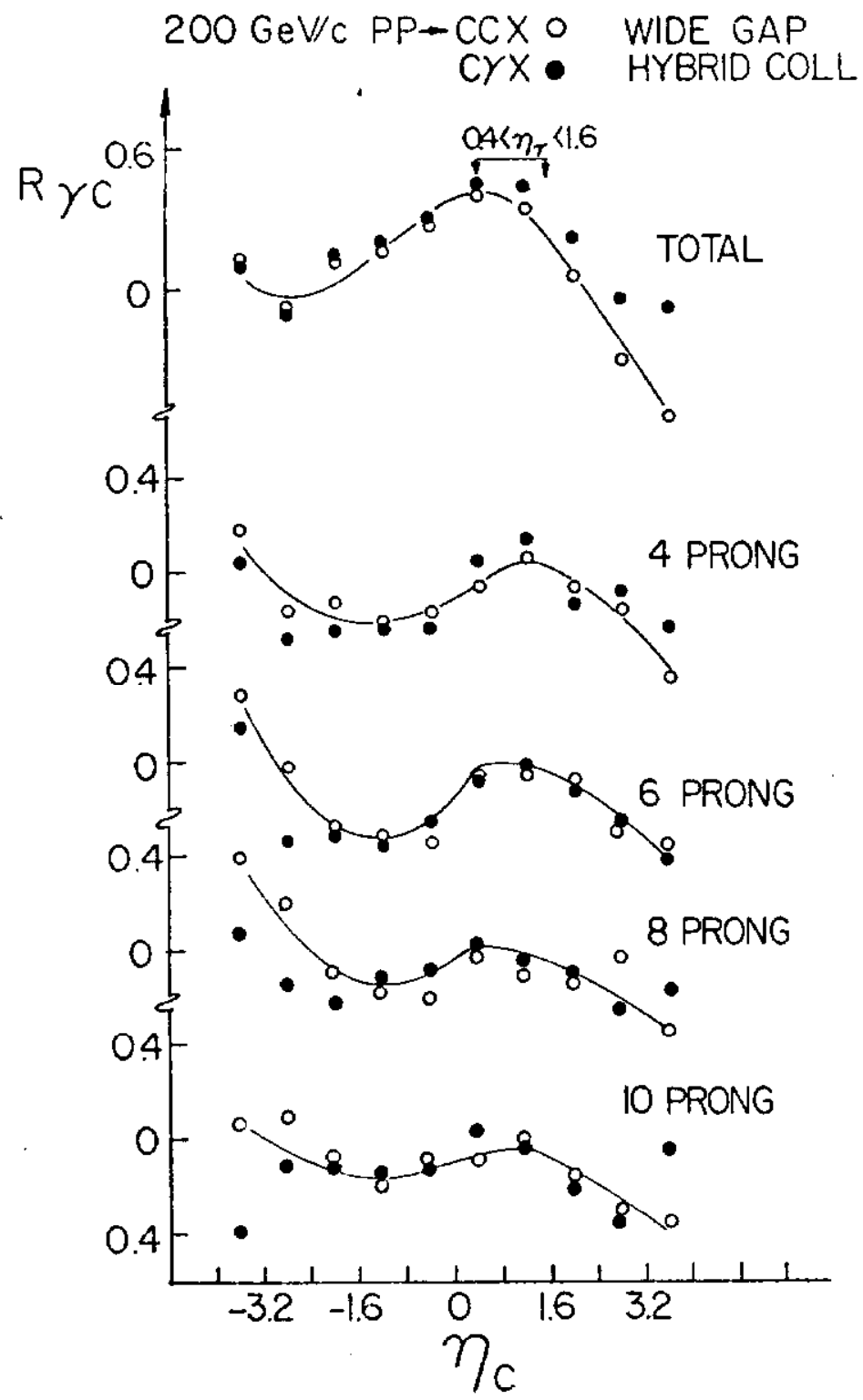

Fig. 15

their original graph, together with the recent $205 \mathrm{GeV} / \mathrm{c}$ data points. There are other interesting aspects to this azimuthal correlation, especially in regard to the different charged particle combinations. 20

In general, correlation study will remain for some time a question of kinematics versus dynamics. On one hand, popular models involving cluster formation can explain many, if not all, of the correlation data gathered so far. But evidences for cluster formation itself have always been circumstantial. On the other hand, there are evidences for 


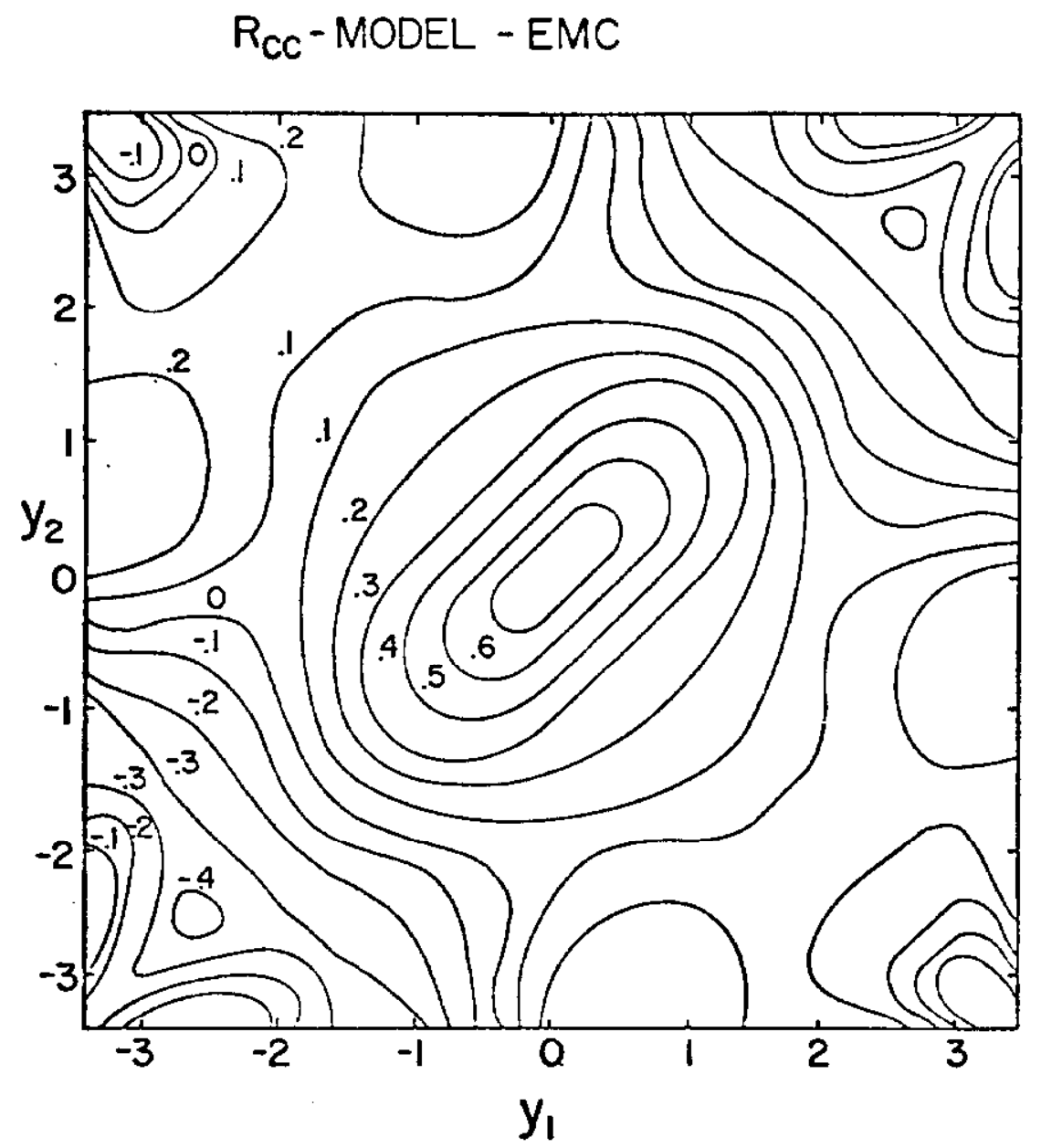

Fig. 16

kinematical reasons arising largely from the energy-momentum conservation and the interplay of a diffractive and a nondiffractive component in the collision process. It appears to me that a deeper understanding in the correlation phenomena requires (a) an experimental effort in separating these two (or perhaps more) components and (b) a careful study of the single-particle inclusive spectra of both the leading and the produced particles. 


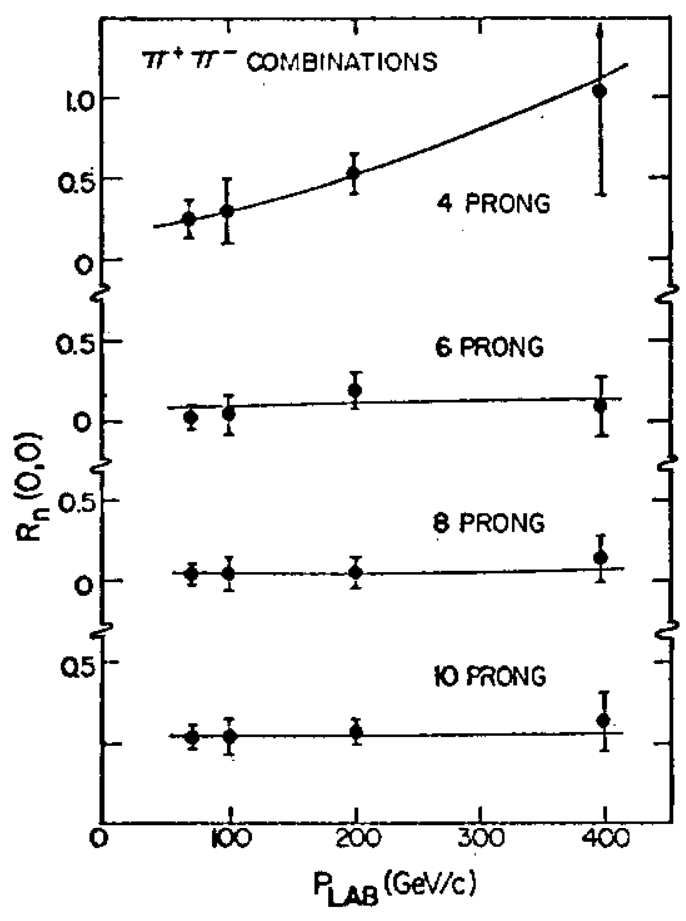

Fig. 17

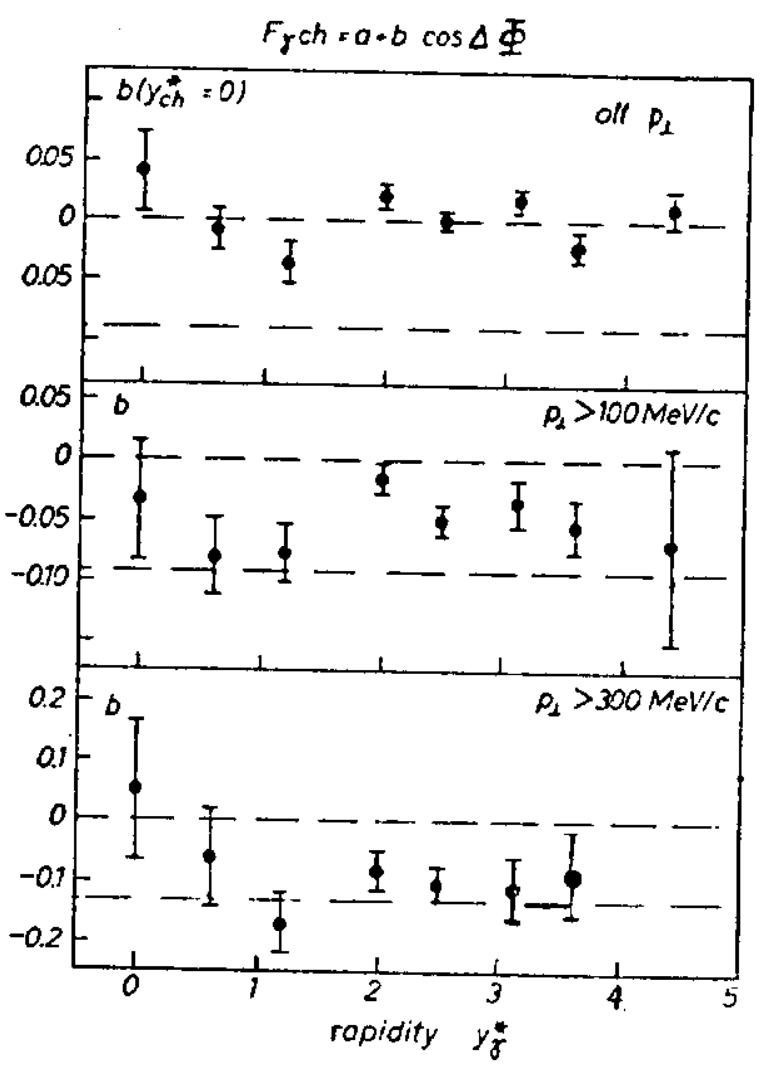



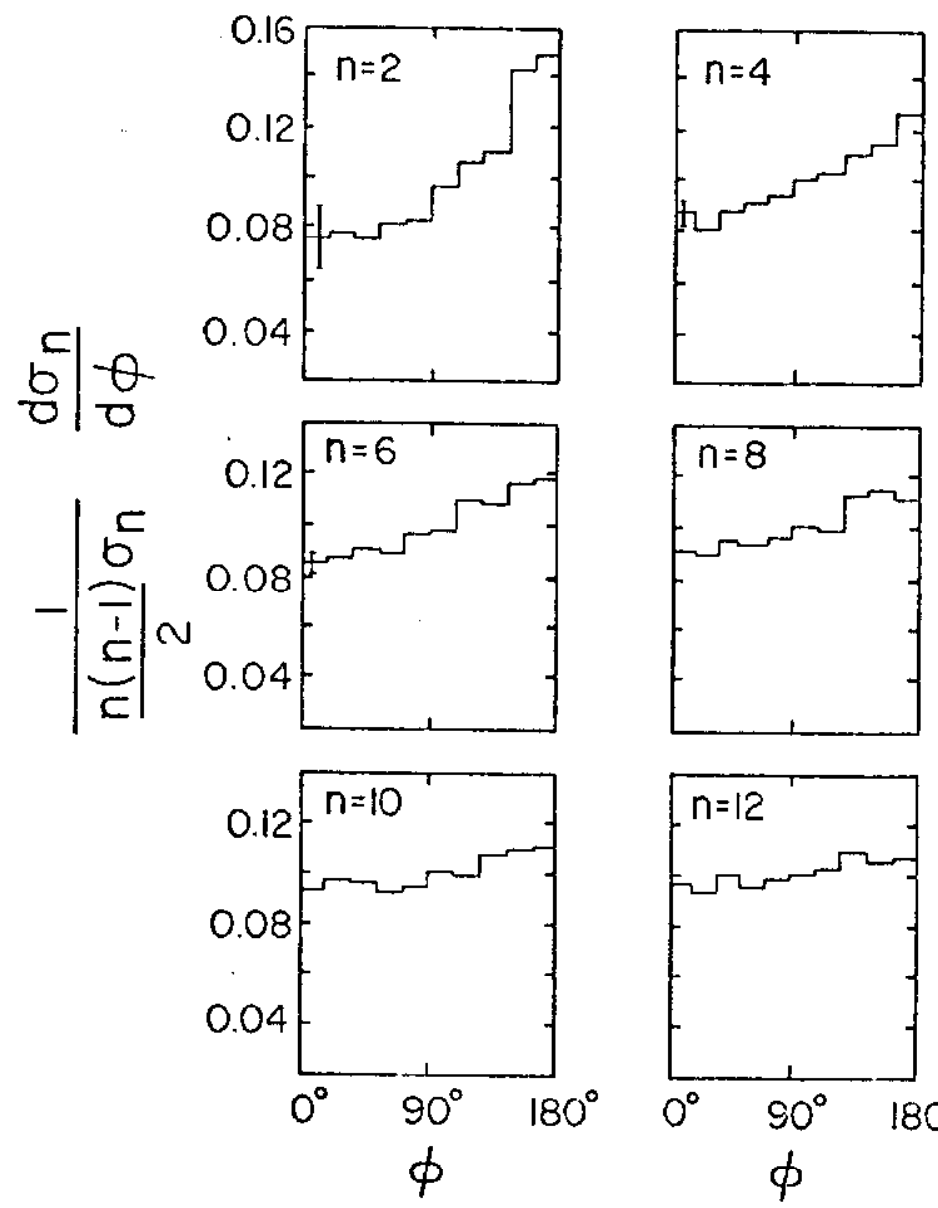

Fig. 19
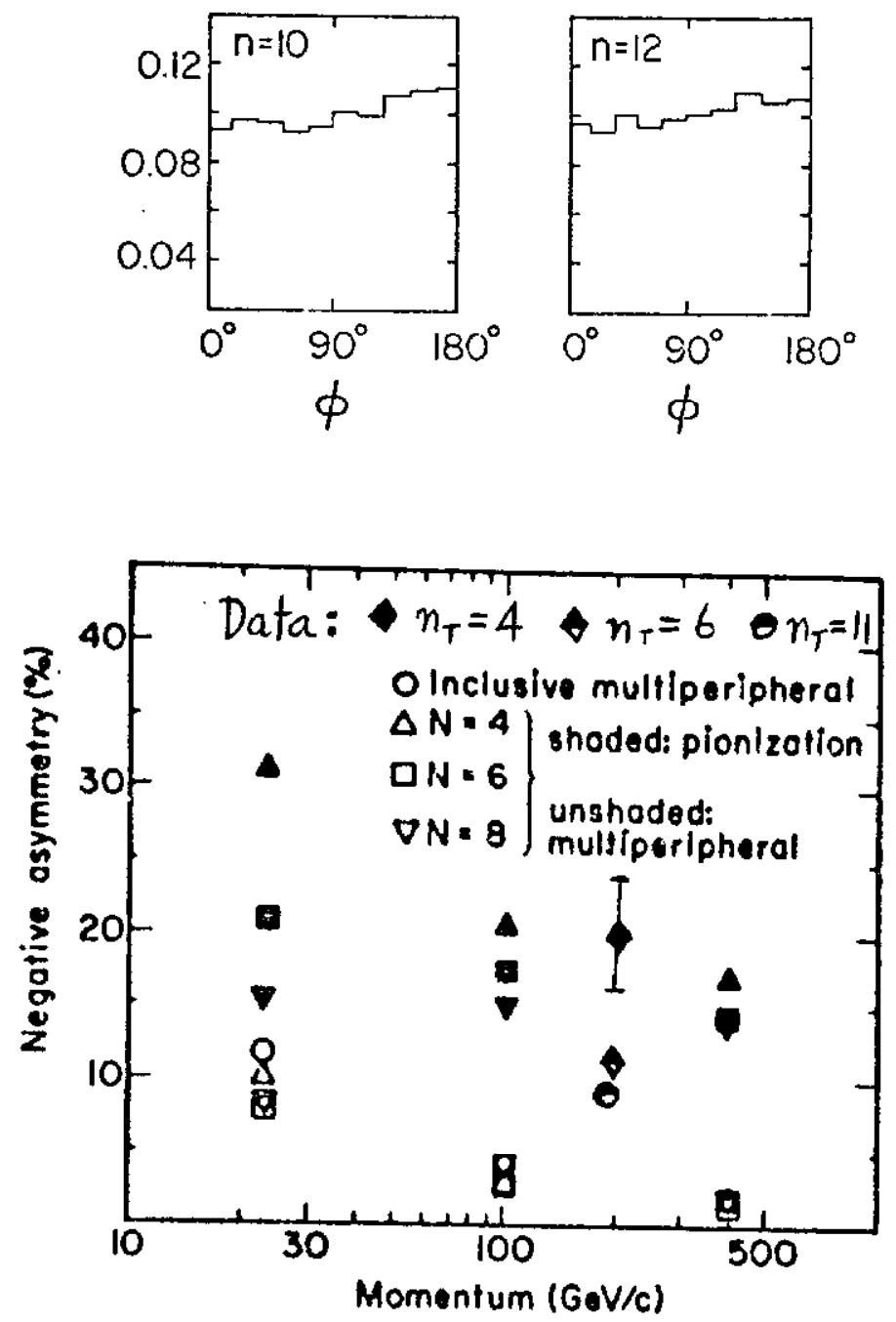

Fig. 20 


\section{SINGLE-PARTICLE INCLUSIVE SPECTRA}

The treatment of single-particle inclusive spectra can best be illustrated in a Peyrou plot as shown in Fig. 21. There are three variables: transverse momentum $\mathrm{p}_{\mathrm{T}}$, longitudinal momentum $\mathrm{p}_{\mathrm{T}}$, and center-of-mass energy $\sqrt{s}$ which gives the boundary. In view of the fact that no satisfactory model has yet given us a "magic" formula which will describe all the inclusive data at all energies, two guidelines have been given by theorists to help experimentalists organize their data in a reasonable form:

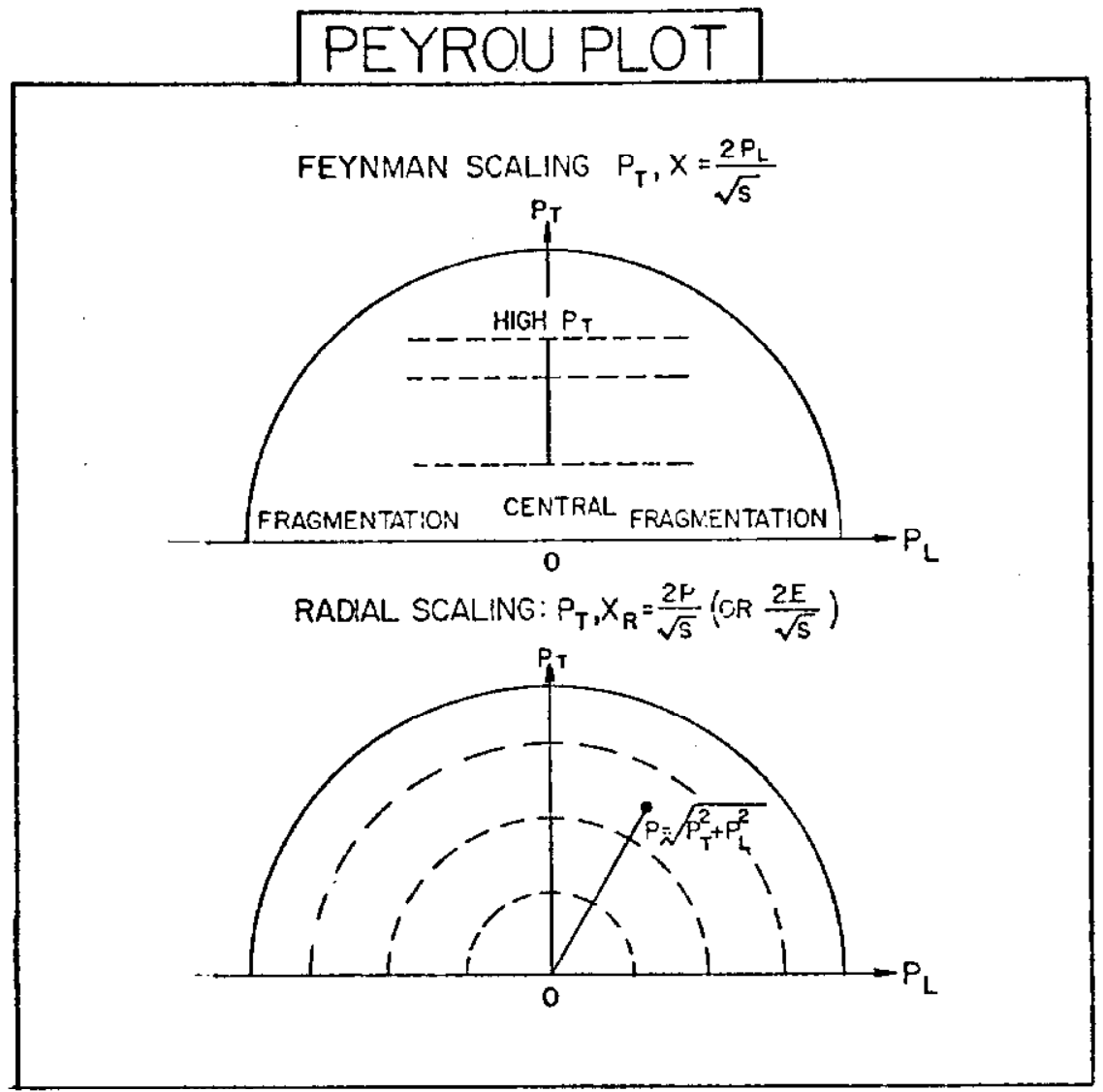

Fig. 21

1. Scaling hypotheses proposed by Yang et al. ${ }^{22}$ and Feynman. 23 In particular the Feynman scaling says

$$
E \frac{d^{3} \sigma}{d p^{3}} \underset{s \rightarrow \infty}{\longrightarrow} f\left(x, p_{\perp}\right)
$$


where

$$
\mathrm{x}=\frac{2 \mathrm{p}_{\mathrm{L}}}{\sqrt{\mathrm{s}}}
$$

and $f$ has no explicit dependence on incident energy.

2. Identifying various regions in the Peyrou plot (see Fig. 21).

Rather than going through these two topics again, I shall first devote some time in describing a new scaling hypothesis proposed by a group of physicists at Fermilab (Walker, Taylor et al. $)^{24}$ and by Yen. ${ }^{25}$ This new proposal has three features:

a. scales better--in other words, organize the data better

b. unifies the Peyrou plot--make the Peyrou plot more continuous

c. (perhaps most important), revives the idea of central collision of Fermi and others.

Their scaling hypothesis (which I shall name the radial scaling) says:

$$
E \frac{d^{3} \sigma}{d p^{3}} \underset{s \rightarrow \infty}{\longrightarrow} f\left(x_{R}, p_{1}\right),
$$

where

$$
x_{R}=\frac{2 \sqrt{p_{L}^{2}+p_{T}^{2}}}{\sqrt{s}} \quad\left(\text { or }=\frac{2 \sqrt{p_{L}^{2}+p_{T}^{2}+M^{2}}}{\sqrt{s}}\right) \text {. }
$$

Walker et al. ${ }^{24}$ further asserts that the factorizability of

$$
f\left(x_{R}, p_{1}\right)=f_{1}\left(x_{R}\right) f_{2}\left(p_{\perp}\right)
$$

We shall examine these two proposals in some detail and make comparison with the Feynman scaling.

(a) Radial Property

The first qualitative difference between the "popular" view and the new proposal is shown in Fig. 21. I believe that this is a matter of emphasis on $\mathrm{p}_{\mathrm{T}}$. If we emphasize the well-known damping in $\mathrm{p}_{\mathrm{T}}$, then the $p_{T}$ values define different regional properties (low $p_{T}$ bremstrahlung and high $\mathrm{p}_{\mathrm{T}}$ parton, etc.) If the damping in $\mathrm{p}_{\mathrm{T}}$ is not emphasized first, 
then we have the idea of central collision with regional properties defined radially. Of course, the whole central region will be affected by the universal damping in $\mathrm{p}_{T}$, but the important point is that the radial properties will then be maintained just like in a conformal mapping.

Figure 22 shows the particle-antiparticle ratios from the two extremes of the Peyrou plot. The data are taken from two recent counter experiments performed at Fermilab. 26,27 The figure shows the patterns of particle-antiparticle ratios are very similar. There are excess of positive particles in both large $p_{T}$ and large $x$. This is probably due to the leading particle effect which may also account for the fact that the difference in positive-to-negative ratio is more significant in the $\mathrm{x}$ direction.

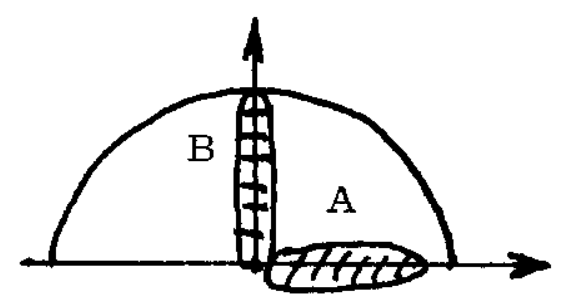

Region A

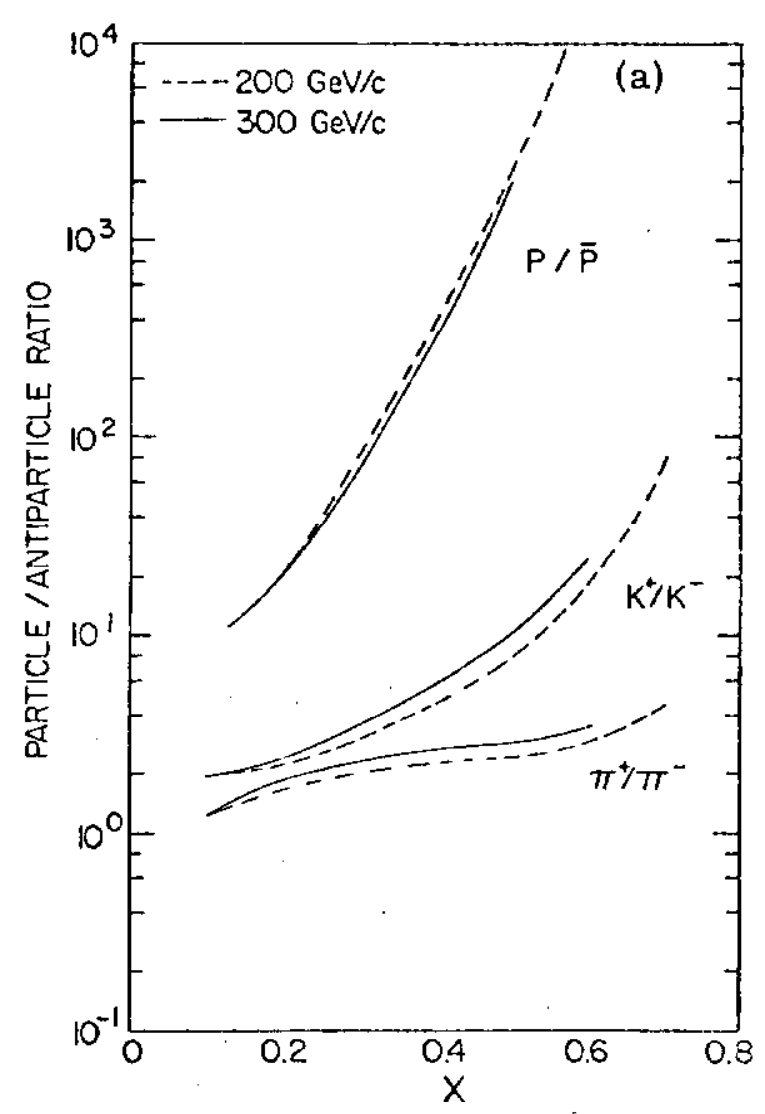

Region B

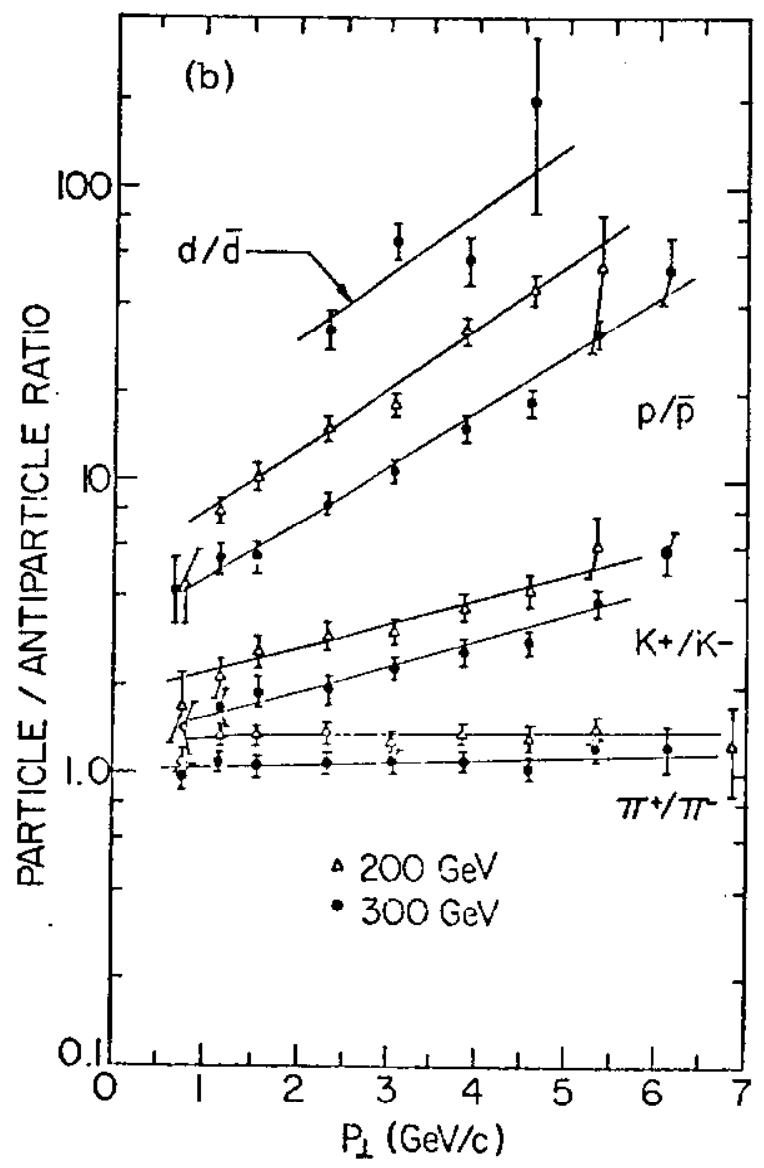

Fig. 22 
Figure 23 shows the particle-to-pion ratios from the same two experiments. Here again the similarity in the two distributions is noted.

Region A

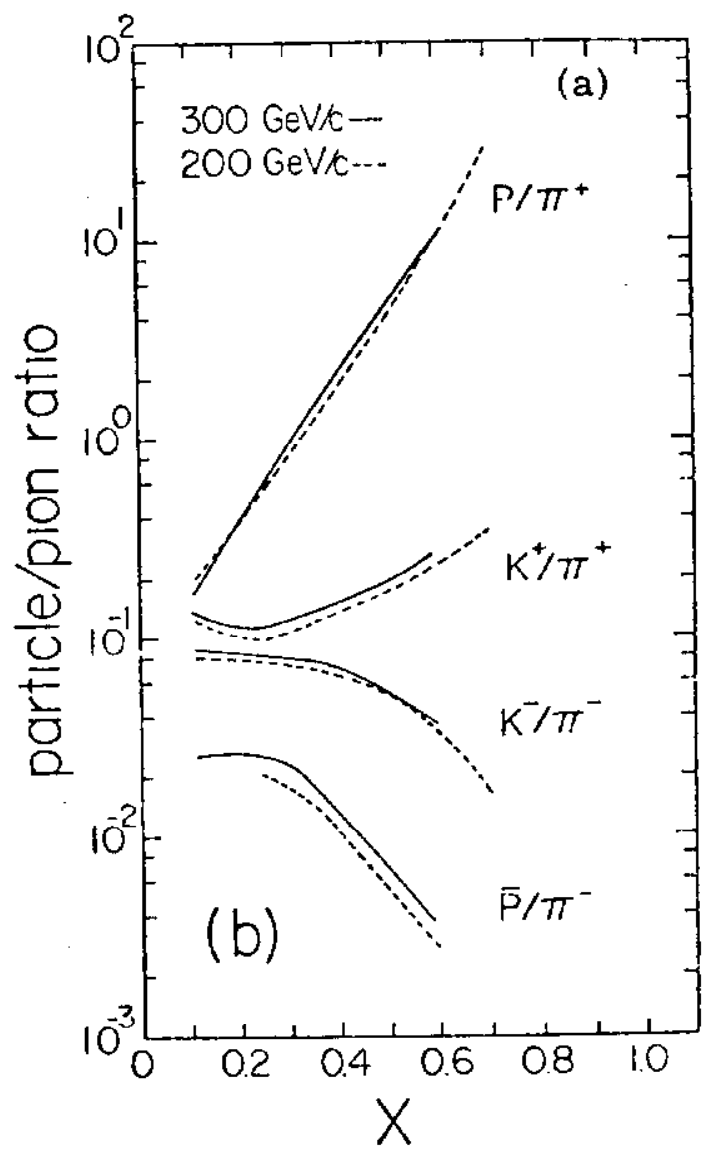

Region $\mathrm{B}$

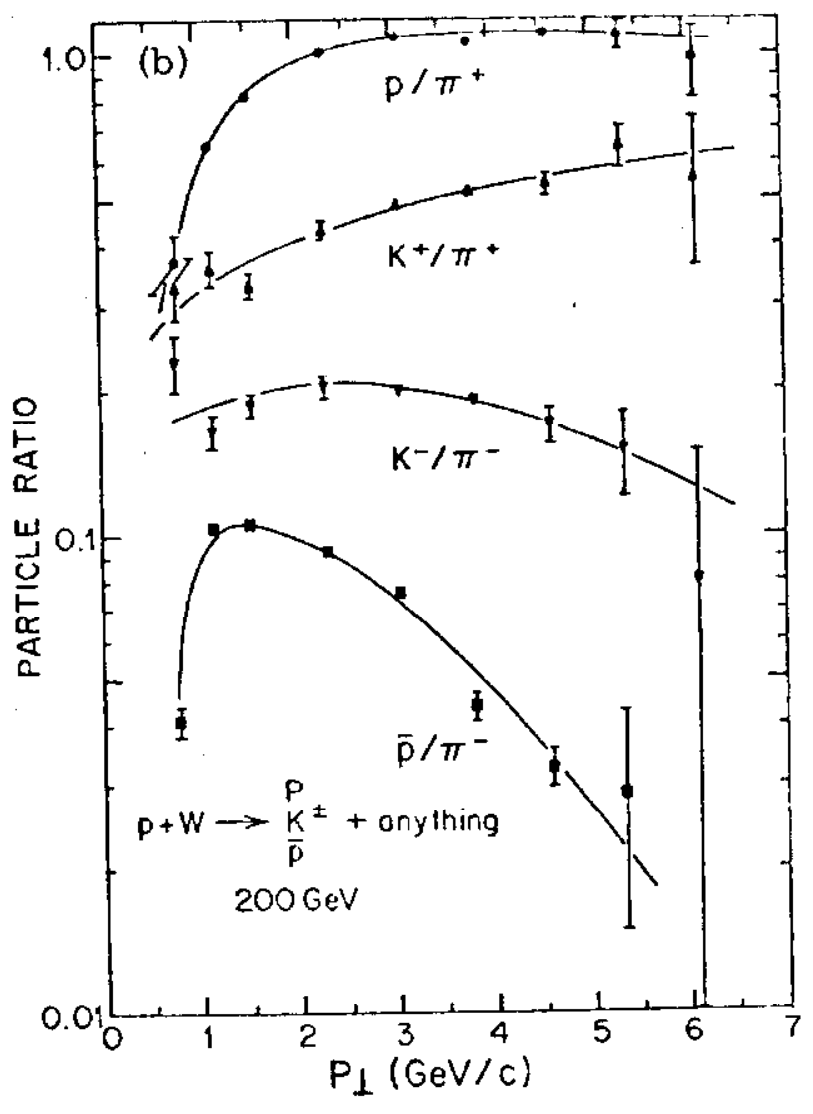

(b) Radial Scaling

Walker et al. first showed that the inclusive reaction $\mathrm{pp} \rightarrow \pi^{\mathrm{O}}+\mathrm{X}$ obeys the radial scaling plus factorization, namely

$$
\left(E \frac{d^{3} \sigma}{d p^{3}}\right)_{\pi} 0=f\left(x_{R}\right) g\left(p_{\perp}\right) .
$$

The detail of their experiment is given in a contributed paper submitted to this conference. 24 They actually worked on the reaction $p p \rightarrow \gamma+X$ at Fermilab over an incident energy from 50 to $400 \mathrm{GeV} / \mathrm{c}$ and six laboratory angles from $30 \mathrm{mrad}$ to $120 \mathrm{mrad}$. (This covered a centerof-mass angle between $20^{\circ}$ and $115^{\circ}$ and transverse momentum from $0.3 \leq \mathrm{p}_{1} \leq 4.3 \mathrm{GeV} / \mathrm{c}$.) They derived the $\pi^{\circ}$ invariant cross section 
using Sternheimer's prescription and assuming that all gammas come from $\pi^{\circ} \rightarrow 2 \gamma$. Figure $24(a)$ shows the radial function $f\left(x_{R}\right)$ for various $\theta_{1 \mathrm{ab}}$ and $\mathrm{p}_{1}$ values. The scaling in the radial function is obvious. Figure $24(\bar{b})$ shows the $g\left(p_{1}\right)$ function and again there is evidence for scaling. A global fit assuming the factorized form of Eq. (9) has been done to check the scaling and a reasonable $x^{2} / d f=1.022$ was obtained.

Yen has also given evidence for the radial scaling for charged particles in inclusive reactions. His compilation covers an incident momenta ranging from 12 to $1500 \mathrm{GeV} / \mathrm{c}$ at ISR. 25 Figure 25(a), (b), and $(c)$ show the invariant cross section for $p p \rightarrow \pi^{-} X, p p \rightarrow \pi^{+} X$, and $\mathrm{pp} \rightarrow \mathrm{pX}$ at $\mathrm{p}_{1}=0.2 \mathrm{GeV} / \mathrm{c}$ and $\mathrm{p}_{1}=0.8 \mathrm{GeV} / \mathrm{c}$. The scaling in the radial variable is evident but the factorizability in the $x_{R}$ and $p_{\perp}$ variable is que stionable because the distributions at $p_{1}=0.2$ and 0.8 are not exactly similar. Thus the radial scaling is supported by the present data but the factorizability at best an approximation. Figure 26 summarizes the data for the inclusive charged particle production up to $1500 \mathrm{GeV} / \mathrm{c}$.

\section{(c) Comparison With Feynman Scaling}

A comparison between the radial scaling and the Feynman scaling can best be done by plotting $E \mathrm{~d}^{3} \sigma / d p^{3}$ versus $\sqrt{\mathrm{s}}$ for fixed $\left(x, p_{1}\right)$ in the case of Feynman scaling and for fixed $\left(x_{R}, p_{1}\right)$ in the case of radial scaling. The data should lie on straight lines if either scaling is valid. This global way of plotting has been suggested by Çronin 27 and others. I have used the data compiled by Giacomelli et al. ${ }^{28}$ in a paper to be published soon. Figures 27, 28, and 29 show that within the scanty data, the radial variable is indeed a better variable. Furthermore, the concept of scaling and the approach to scaling depends very much on the selected variable.

For initial states other than pp interactions, no symmetry is expected between the "target" and the "beam" hemispheres. The radial distribution is likely to differ in the two hemispheres. Speculations (??) on the single-particle inclusive distributions in $\pi^{-} p, K^{+} p, \bar{p} p$, and $e^{+} e^{-}$ $(\rightarrow$ hadrons) interactions are given in Fig. 30. Ongoing experiments at Fermilab and elsewhere will soon show whether these guesses are intelligent. 


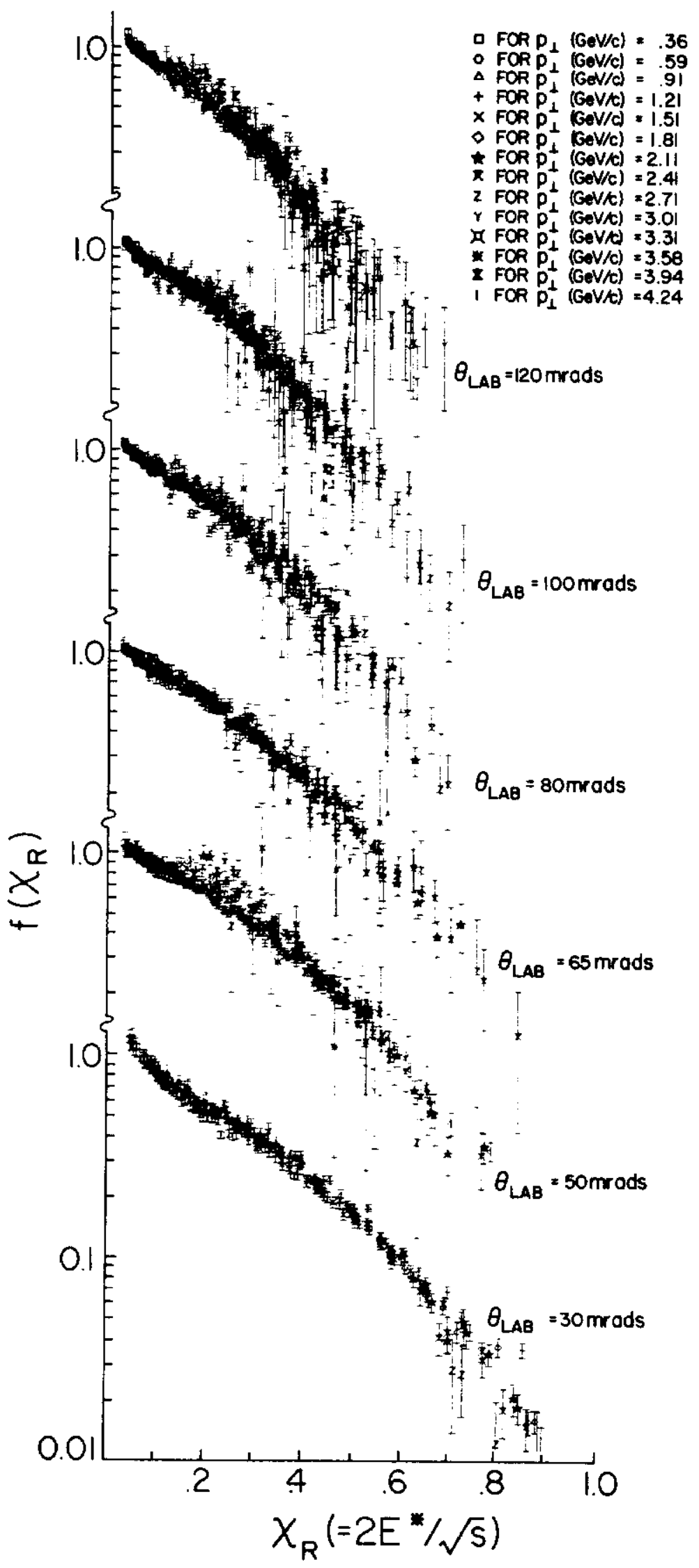

Fig. 24(a) 


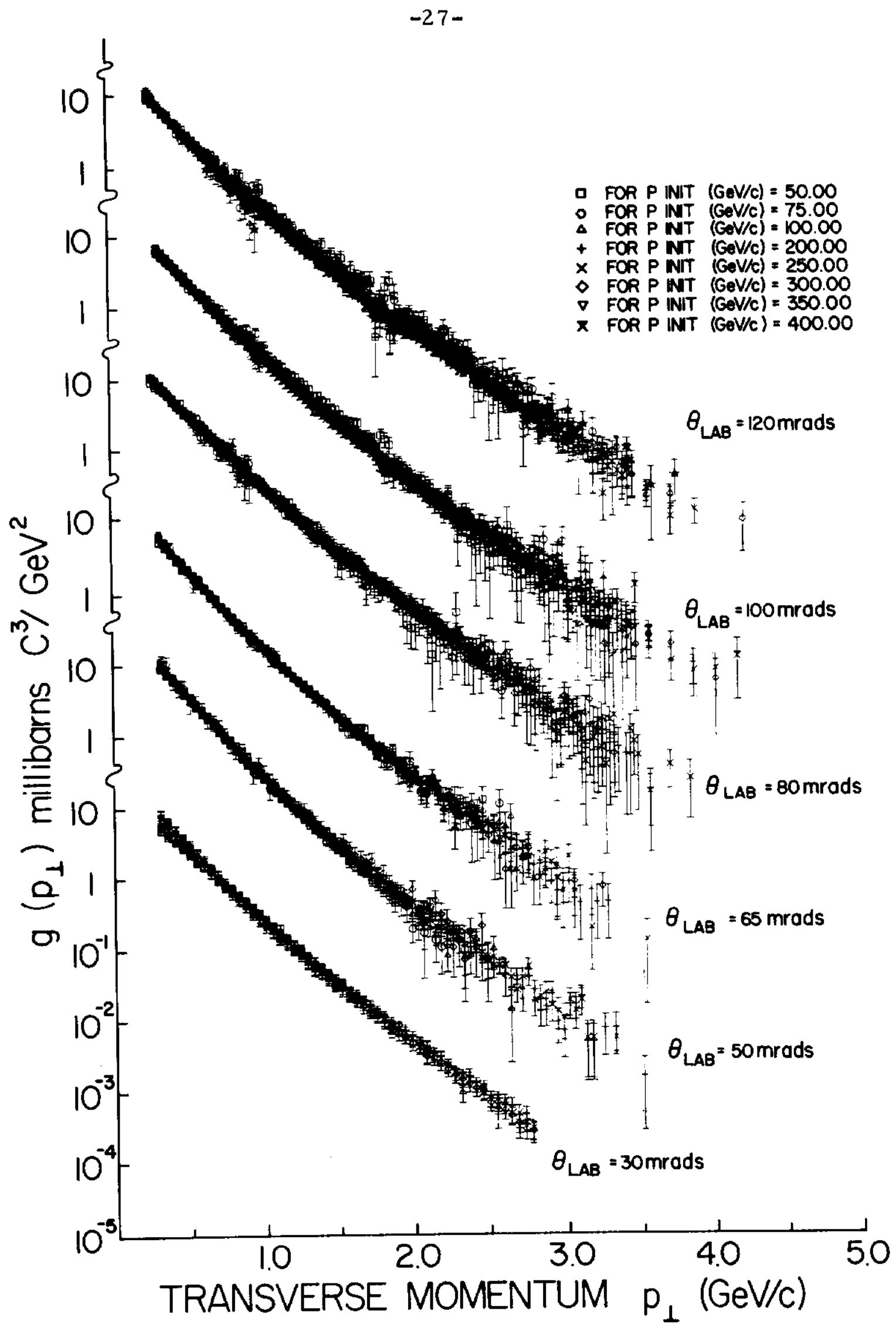

Fig. $24(b)$ 
$-28-$

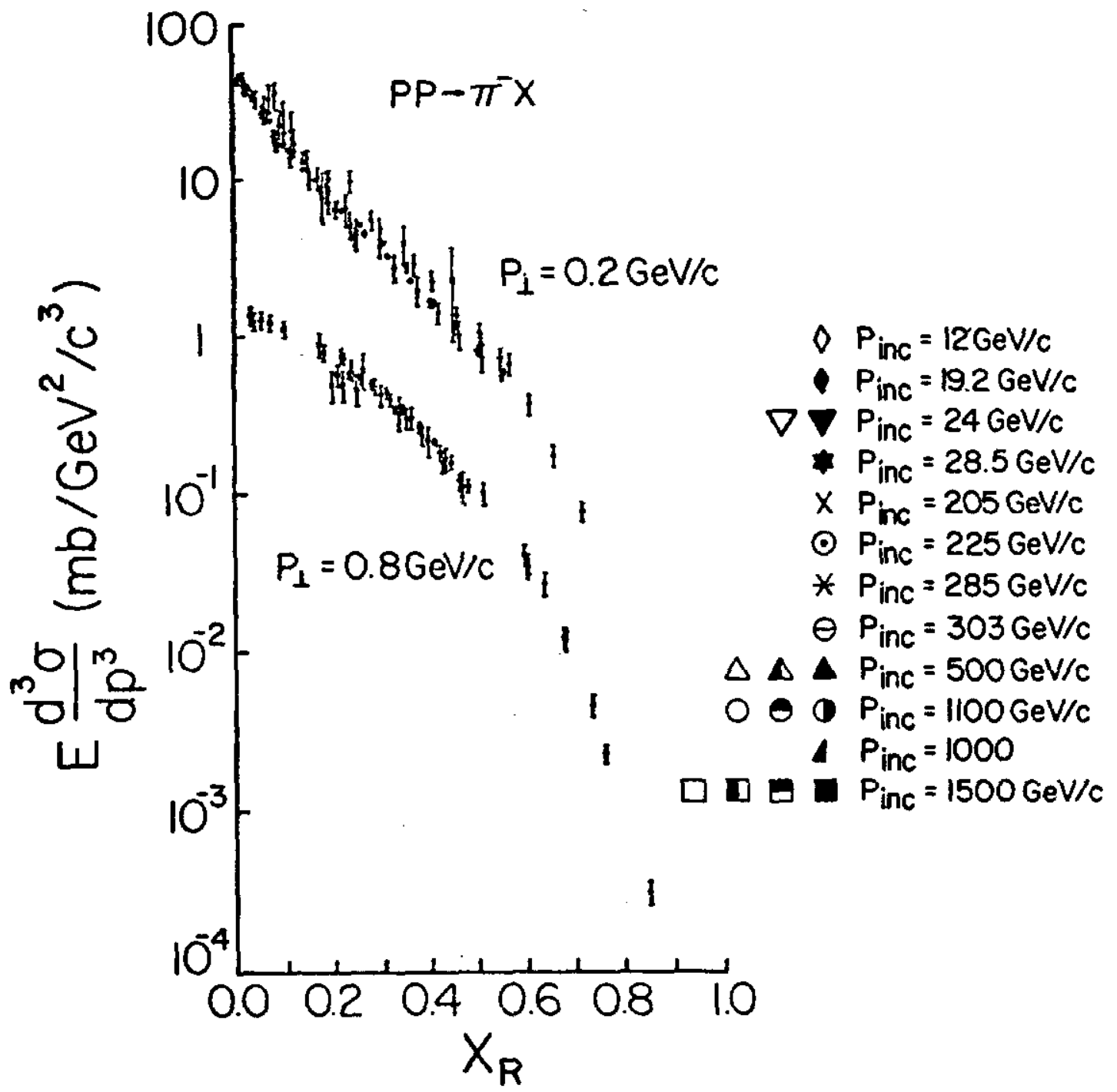

Fig. 25(a) 
$-29-$

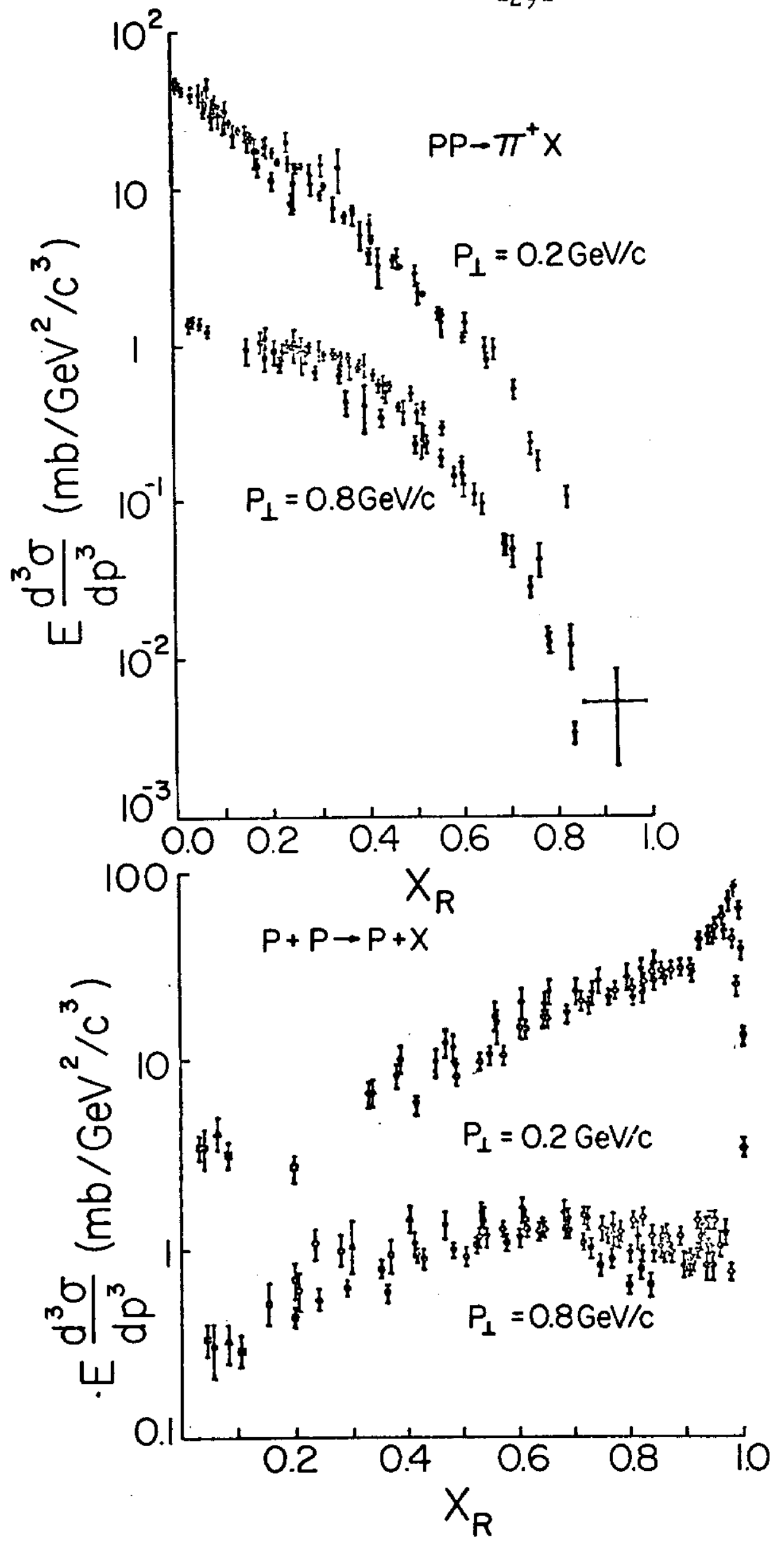

Fig. 25(b)

Fig. 25(c) 


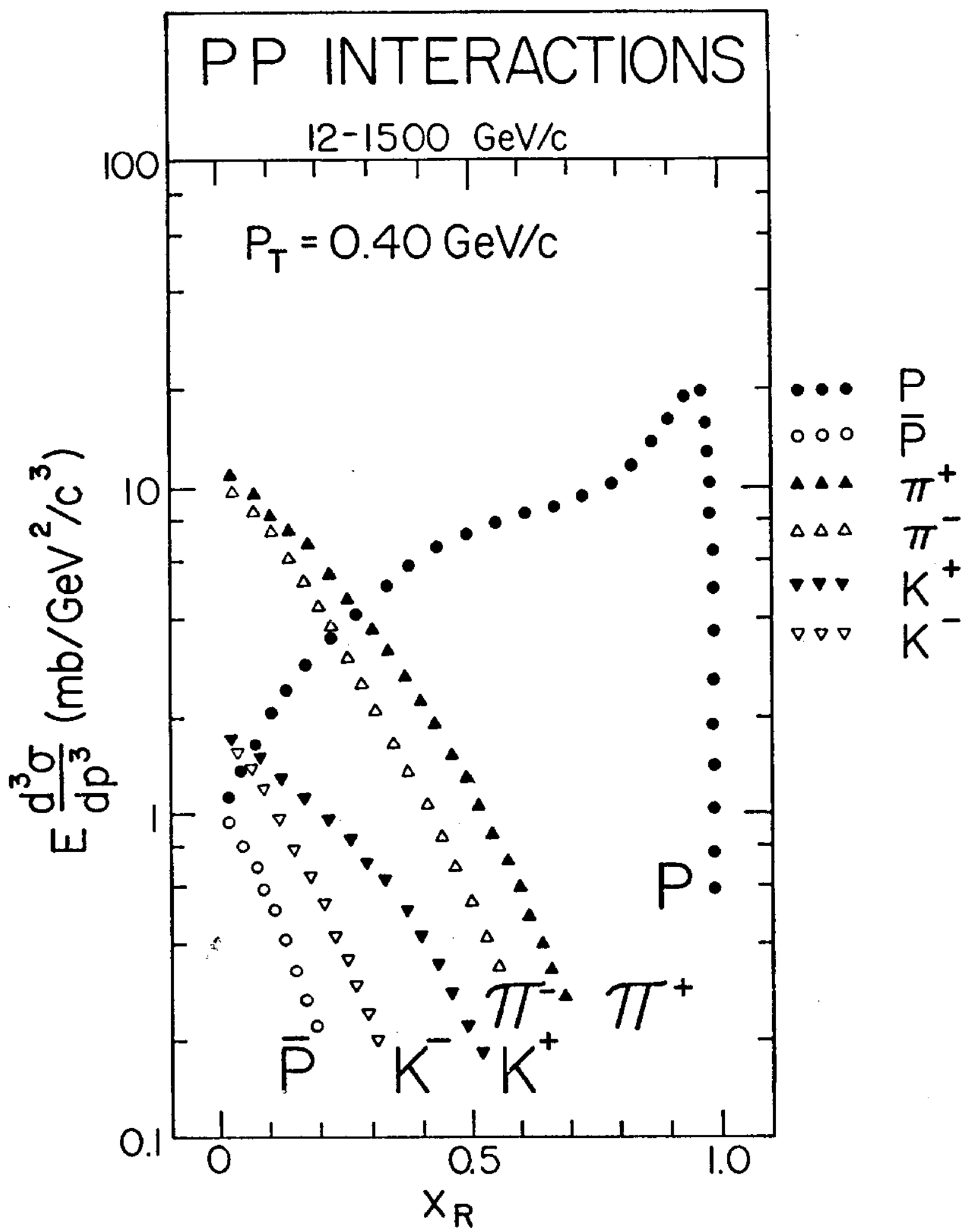

Fig. 26 
$-31-$

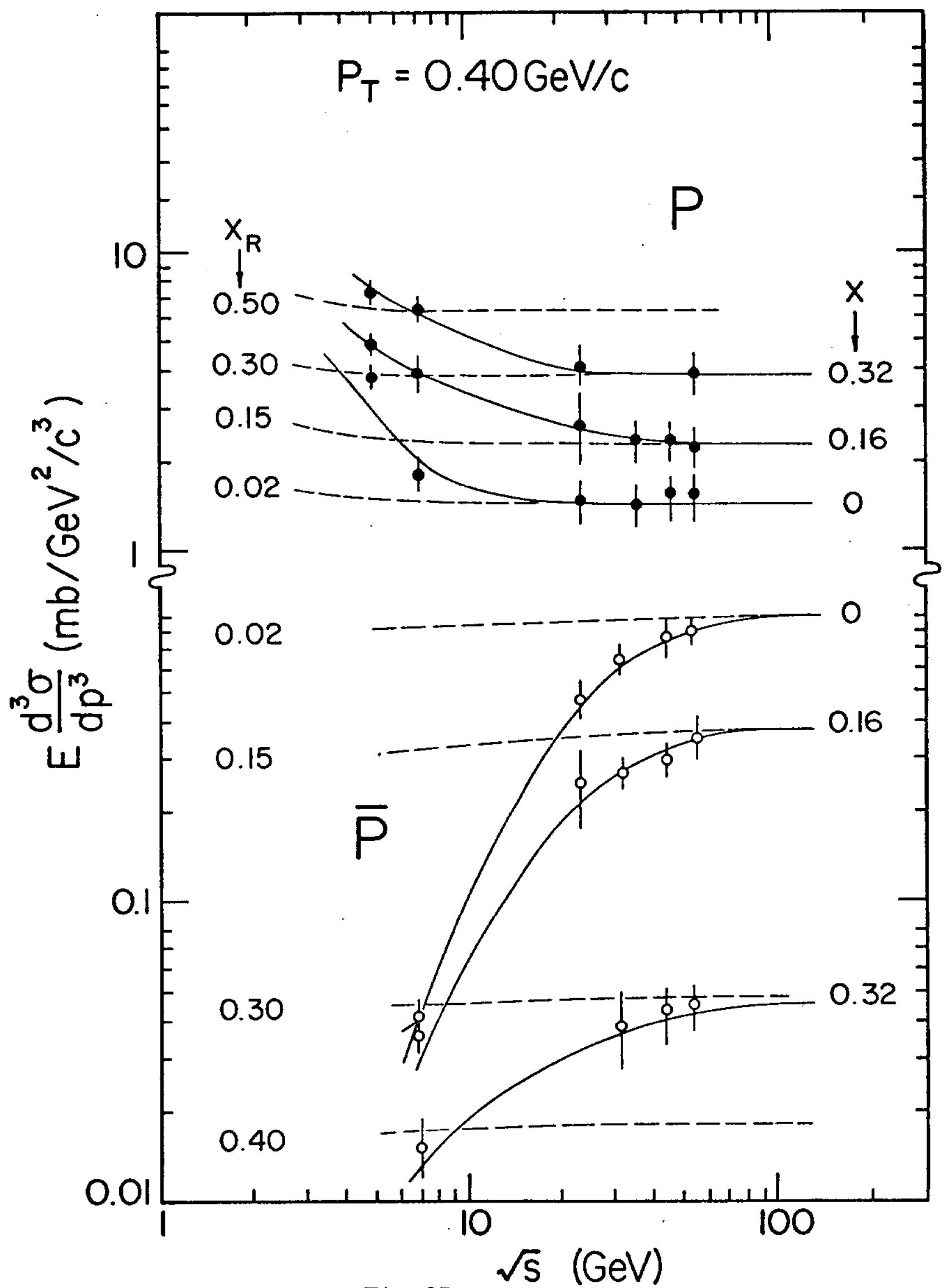

Fig. 27 


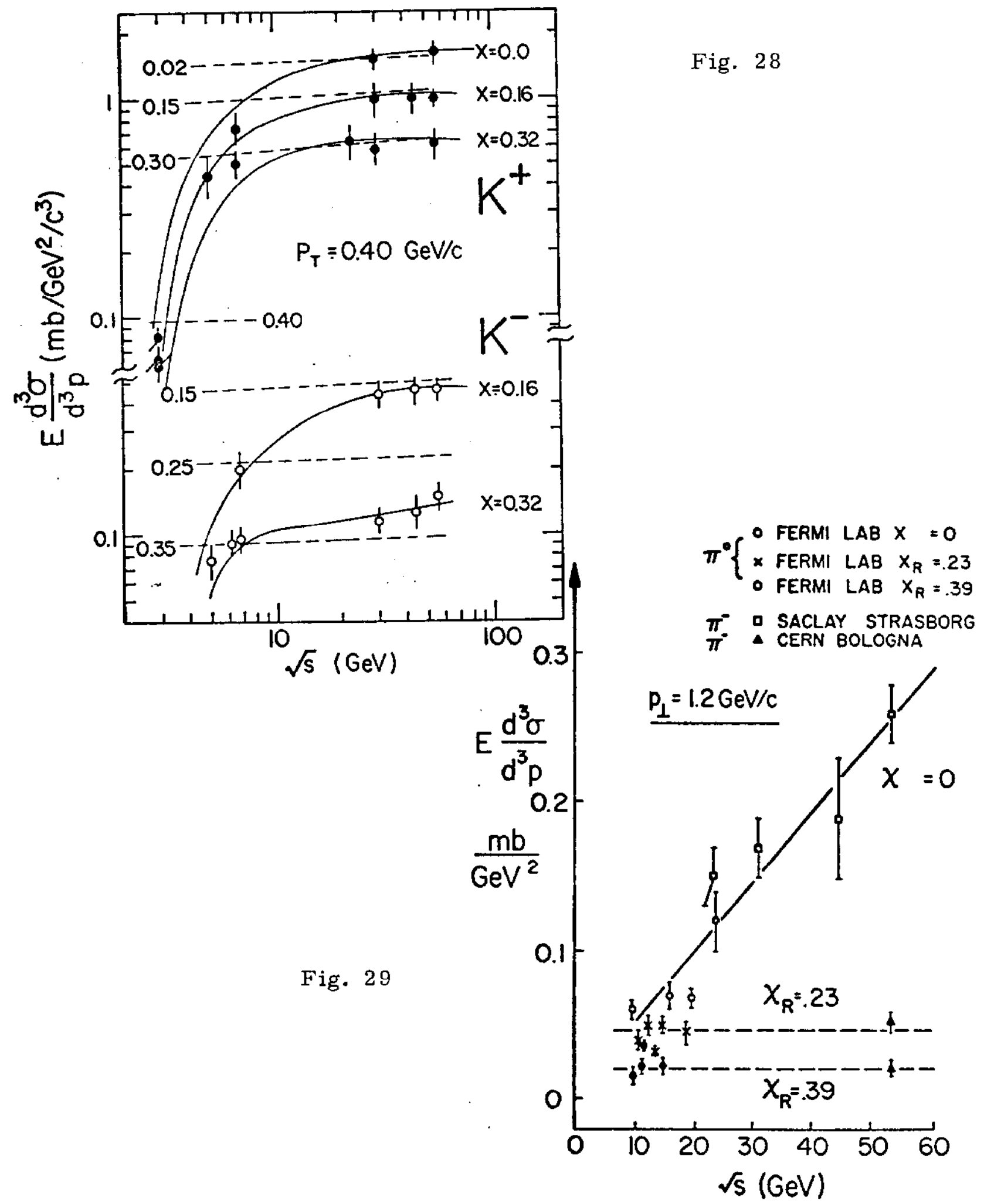



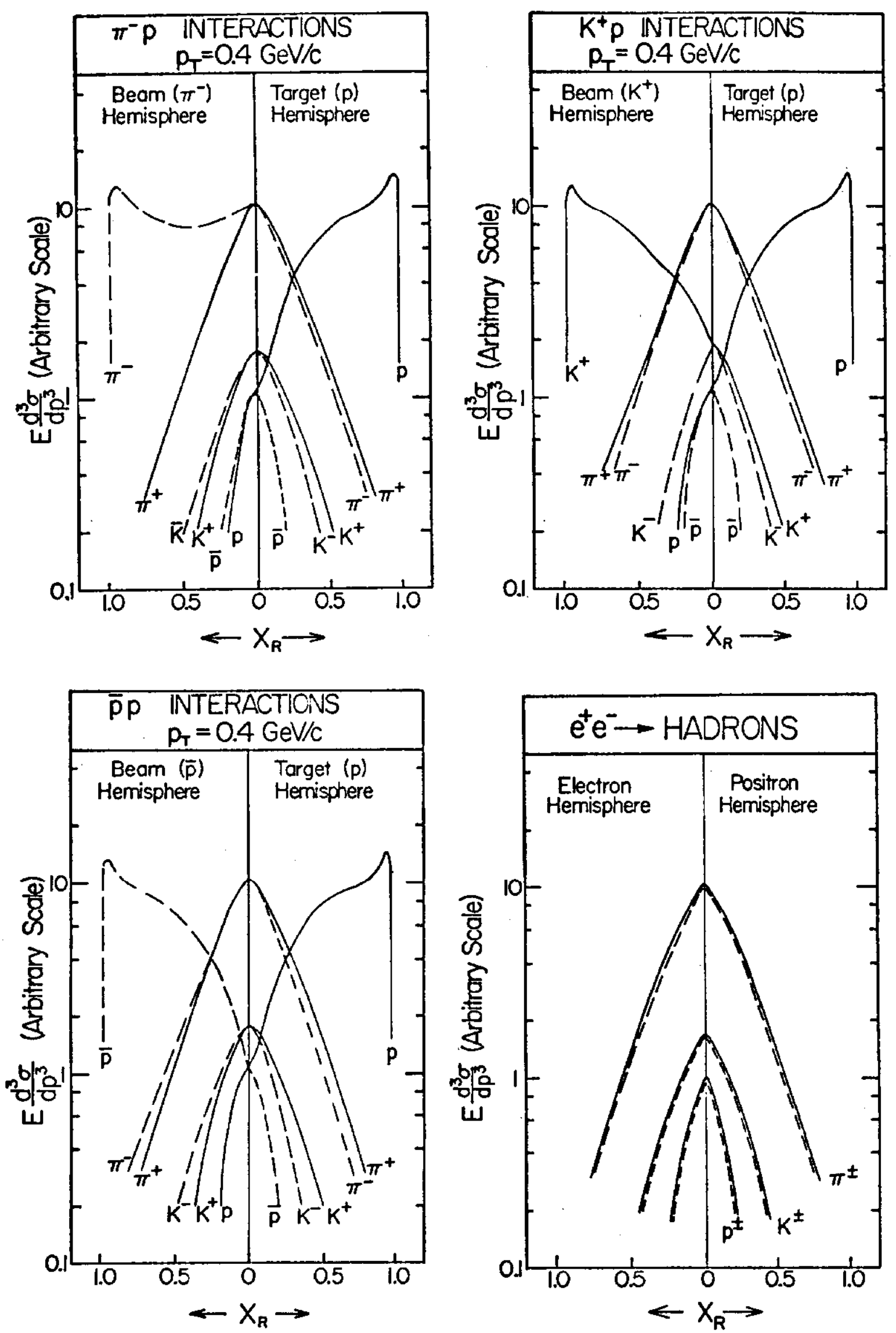

Fig. 30 


\section{CONCLUSION}

The following table gives a summary of this review and some suggestions for further research.

\begin{tabular}{|c|c|c|}
\hline Topic & Summary & Further Research \\
\hline Multiplicity & $\begin{array}{l}\text { Charged and associated } \\
\text { multiplicities show simple } \\
\text { systematics: (a) slow } \\
\text { increase }<n_{c}>\mathrm{m} \text { s and } \\
\text { (b) shape of distribution } \\
\text { independent of } \sqrt{\mathrm{s}} \text { and } \\
\text { initial state. }\end{array}$ & $\begin{array}{l}\text { To determine neutral } \\
\text { and charged multiplicity } \\
\sigma\left(n_{C}, n_{O}\right) ; \text { multiplicity } \\
\text { composition, i. e. } \\
\text { diffractive and non- } \\
\text { diffractive components. }\end{array}$ \\
\hline Correlation & $\begin{array}{l}\text { Major role played by } \\
\text { energy-momentum con- } \\
\text { servation and the inter- } \\
\text { play of diffractive and } \\
\text { nondiffractive com- } \\
\text { ponent does not show } \\
\text { much correlation. }\end{array}$ & $\begin{array}{l}\text { Decomposition into } \\
\text { diffractive and non- } \\
\text { diffractive events and } \\
\text { repeat correlation study. } \\
\text { Re-examine definitions } \\
\text { of } R \text { and } C \text {. }\end{array}$ \\
\hline $\begin{array}{l}\text { Single-particle } \\
\text { inclusive }\end{array}$ & $\begin{array}{l}\text { Radial scaling sum- } \\
\text { marizes data better; } \\
\text { radial properties has } \\
\text { also been observed in } \\
\text { particle-to-antiparticle } \\
\text { composition; concept of } \\
\text { scaling and approach to } \\
\text { scaling depends on } \\
\text { variables selected. }\end{array}$ & $\begin{array}{l}\text { More experiments on } \\
\pi p, K p \text {, and } \bar{p} p ; \text { study } \\
\text { leading particle effects } \\
\text { and dimensionality of } \\
\text { collision process. }\end{array}$ \\
\hline
\end{tabular}

\section{ACKNOWLEDGMENT}

I would like to thank the chairman Bob Panvini for inviting me and helping me prepare this review talk. My gratitude to Ernie Malamud, Jim Whitmore, and Frank Taylor for reading the first draft and giving valuable comments. Discussions with many people especially Chris Quigg and G. Giacomelli have convinced me that the field of strong interaction is still fresh and exciting. Finally, my indebtedness to all groups and experimentalists who have contributed papers and graphs to this conference. 


\section{REFERENCES}

1 J. Whitmore, Physics Reports 10, 274 (1974).

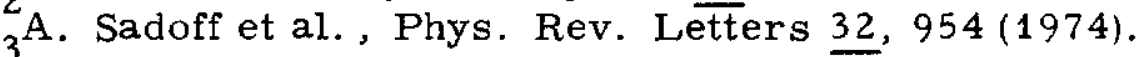

4 Z. Koba, H. B. Nielsen, and P. Olesen, Nucl. Phys. B40, 317 (1974).

${ }_{5}^{4}$ P. Slattery, Phys. Rev. Letters 29, (1972).

A. Wroblewski, Acta. Phys. Polonica B4, 857 (1973); see also

A. Wroblewski, invited talk given at the IVth International Symposium on Multiparticle Hadrodynamics, Collegio Ghislierli Pavia, August 631 -September 4, 1973.

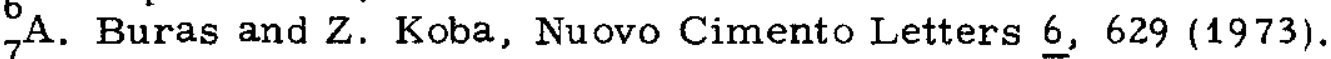

E. Wigner, Proc, of the Conference on Neutron Physics by Time-of${ }_{8}$ Flight, Gatlinburg, Tennessee (1957).

Statistical Theories of Spectra: Fluctuations, C. E. Porter, Editor 9 (Academic Press, N.Y., 1965).

F. Winkelmann, paper presented at the IV ${ }^{\text {th }}$ International Conference on Experimental Meson Spectroscopy, Boston, April 26-27, 1974;

10 preprint LBL-3045 (1974).

$11_{\mathrm{R}} \mathrm{R}$. Singer, private communication concerning the $205 \mathrm{GeV} / \mathrm{c} \mathrm{pp}$ data. Contribution to this converence from the wide-gap spark chamber collaboration of ISU-MSU-MD-ANL-FNAL. Data furnished by

12 G. Smith of Michigan State University.

13 F. T. Dao and J. Whitmore, Phys. Letters B46, 252 (1973).

F. T. Dao, J. Lach, and J. Whitmore, Fermi National Accelerator Laboratory Report NAL-Pub-73/81-EXP, to be published in Phys. Letters. See also T. Kitagaki, paper presented at the International Conference on Antinucleon Interactions, Liblice, Czechoslovakia, 14 June 23-28, 1974.

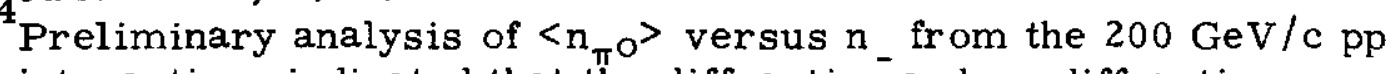
interactions indicated that the diffractive and nondiffractive components do not show a strong linear correlation between $\pi^{\circ}$ and $\pi^{-}$, as demonstrated in the total inclusive data of Fig. 7. Private communi15 cation from Karl Jaeger and Jim Whitmore.

${ }^{\mathrm{W}}$. Ko, review paper presented at the XVII International Conference

16 on High Energy Physics, London, July 1974; preprint UCD-PPL-7-17-74.

${ }_{17}^{16}$ R. Singer et al. , Phys. Letters B49, 481 (1974).

V. V. Babintsev et al. Abstract number 456, paper submitted to the

XVII International Conference on High Energy Physics, London,

18 July 1974.

R. Singer et al. , Argonne National Laboratory Report ANL/HEP -7445

(1974). This is a preliminary version, and I would like to thank

19 R. Singer for the communication.

CERN-Hamburg-Wienna Collaboration, paper number 338 submitted to the $2^{\text {nd }} \mathrm{Aix}$-en-Provence International Conference on Elementary Particles, France, September 1973. 
${ }^{20}$ M. Pratap et al. , Azimuthal Correlations in pp Interactions at 205 $\mathrm{GeV} / \mathrm{c}$, Stony Brook-Argonne-Fermilab Collaboration, to be published 21 in Phys. Rev. Letters (1974).

J. H. Fredman, C. Risk, and D. B. Smith, Phys. Rev, Letters 28 , $22^{191(1972) \text {. }}$

23 J. Benecke et al. , Phys. Rev. 188 (1969).

${ }_{24}$ R. P. Feynman, Phys. Rev. Letters 23, 1415 (1969).

D. C. Carey et al., Inclusive $\pi^{\circ}$ Production in pp Collisions at 50-400 $\mathrm{GeV} / \mathrm{c}$, paper submitted to this conference. See also references by the same authors in Phys. Rev. Letters 32, 24 (1974) and Phys. Rev. ${ }_{25}$ Letters 33,327 (1974).

E. Yen, $\bar{N}$ ew Scaling Variable and Early Scaling in Single Particle Inclusive Particle Inclusive Distributions for Hadron-Hadron Collisions, Michigan State University preprint (1974).

${ }^{26}$ W. F. Baker et al. , Measurement of $\pi^{ \pm}, K^{ \pm}, P$ and $\bar{P}$ Production by 200 and $300 \mathrm{GeV} / \mathrm{C}$ Protons, Fermi National Accelerator Laboratory

27 Report NAL-Pub-74/13-EXP, to be published in Phys. Letters. J. W. Cronin, Processes at Large Transverse Momentum, talk prepared for the SLAC Summer Institute on Particle Physics, August 10,1974 . I would like to thank C. Quigg for giving me a preprint of ${ }_{28}$ Cronin's talk.

A. M. Rossi, G. Giacomelli et al. , Experimental Study of the Energy Dependence in Proton-Proton Inclusive Reactions, paper submitted to Nucl. Phys. I would like to thank G. Giacomelli for giving me a preliminary version of this paper. 


\section{FIGURE CAPTIONS}

Fig. 1. The average charged particle multiplicities per inelastic collision as a function of laboratory momentum. The solid and dashed curves are fits to pp data: $\left\langle\mathrm{n}_{\mathrm{c}}\right\rangle=-4.8+10 \mathrm{~s}^{-\frac{1}{2}}+2.0 \mathrm{ens}$ and $\left\langle\mathrm{n}_{\mathrm{C}}\right\rangle=-2.9+1.79 \ln \mathrm{s}$.

Fig. 2. Average and associated hadron multiplicity in system $X$ as a function of c.m. energy squared.

Fig. 3. Plot of $\langle n\rangle \sigma_{n} / \sigma_{\text {in }}$ versus $n /\langle n\rangle$ for (a) $\pi^{-}$p interactions and (b) $\mathrm{pp}$ interactions.

Fig. 4. Distribution of level spacings in excited states from (a) atomic spectra and (b) nuclear spectra. $\langle s\rangle$ is the average level spacing and the probability $\mathbf{P}$ of the distribution has been normalized to 2 .

Fig. 5. Invariant mass distributions of $\pi \pi$ combinations for (a) $205 \pi / p$ $\mathrm{GeV} / \mathrm{c}$ interactions and (b) $205 \mathrm{GeV} / \mathrm{c}$ pp interactions.

Fig. 6. Average number of $\pi^{\circ}$ as a function of the negative particle multiplicity.

Fig. 7. Average number of $\pi^{\circ}$ as a function of the negative particle multiplicity for $\mathrm{pp}, \pi \mathrm{N}$, and $\overline{\mathrm{p}}$ interactions. The $\alpha^{\prime} \mathrm{s}$ are obtained from fitting the data to $\left\langle\mathrm{n}_{\mathrm{o}}\right\rangle=\alpha \mathrm{n}_{-}+\beta$.

Fig. 8. Average number of $\mathrm{K}_{\mathrm{S}}{ }^{\circ}$ and $\Lambda$ per inelastic pp collision as a function of the negative particle multiplicity.

Fig. 9. Two-particle Muller correlation parameter as a function of laboratory momentum for (a) $\mathrm{K}_{\mathrm{S}}{ }^{\mathrm{O}}$ and $\pi^{-}$pairs and (b) $\Lambda^{\circ}$ and $\pi^{-}$ pairs.

Fig. 10. Average number of $\pi^{\circ}$ as a function of the negative particle multiplicity from $15 \mathrm{GeV} / \mathrm{c}$ pp interactions for (a) all inelastic events and (b) events separated by ionization into annihilation and non-annihilation components.

Fig. 11. Contour plot of two-particle correlation function $R_{c c}\left(y_{1}, y_{2}\right)$ as obtained from $205 \mathrm{GeV} / \mathrm{c}$ pp interactions.

Fig. 12. Two-particle correlation function versus rapidity difference $y_{1}-y_{2}$ for (a) $\pi^{c} \pi^{c}$, (b) $\pi^{+} \pi^{-}$, (c) $\pi^{+} \pi^{+}$, and (d) $\pi^{-} \pi^{-}$combinations.

Fig. 13. Semi-inclusive $\pi^{-} \pi^{-}$rapidity correlation as a function of $y_{2}{ }^{-}$ for fixed $\mathrm{y}_{1}^{-} \approx 0$.

Fig. 14. Semi-inclusive $\pi^{+} \pi^{-}$rapidity correlation as a function of $y_{1}^{+}$ for fixed $\mathrm{y}_{2} \overrightarrow{-} \approx 0$.

Fig. 15. Two-particle inclusive and semi-inclusive rapidity correlation as a function of $\eta_{\mathrm{C}}$ for charged-charged and charged-gamma combinations. The other particle is fixed at $0.4<\eta_{c}\left(\eta_{Y}\right)<1.6$.

Fig. 16. Contour plot of two-particle correlation function $R_{\mathrm{cc}}\left(\mathrm{y}_{1}, \mathrm{y}_{2}\right)$ from Monte Carlo calculation of R. Singer et al., Ref. 18.

Fig. 17. $R_{n}(0,0)$ as a function of laboratory momentum. Lines are drawn to guide the eye.

Fig. 18. Angular parameter $b$ as a function of gamma rapidity for three selections of charged particle transverse momentum. 
Fig. 19. Normalized azimuthal angular distributions for different charged multiplicity.

Fig. 20. Negative asymmetry as a function of laboratory momentum Data are from Ref. 21.

Fig. 21. Schematic diagram of Peyrou plots for Feynman scaling and radial scaling. Dotted lines delineate regions with similar physical properties.

Fig. 22. Particle-to-antiparticle ratios in two kinematic regions as a function of (a) $x$, and (b) $\mathrm{p}_{\mathrm{T}}$.

Fig. 23. Particle-to-pion ratios in two kinematic regions as a function of (a) $x$, and (b) $\mathrm{p}_{\mathrm{T}}$.

Fig. 24. Single-particle inclusive distributions from $\mathrm{pp} \rightarrow \pi^{\circ} \mathrm{X}$ inter actions for (a) radial function versus $x_{R}$ and (b) transverse function versus $\mathrm{p}_{\mathrm{T}}$.

Fig. 25. Single-particle radial distributions at $\mathrm{p}_{\mathrm{T}}=0.2$ and 0.8 for (a) $\mathrm{pp} \rightarrow \pi^{-} \mathrm{X}$, (b) $\mathrm{pp} \rightarrow \pi^{+} \mathrm{X}$, and (c) $\mathrm{pp} \rightarrow \mathrm{pX}$.

Fig. 26. Single-particle radial distributions for charged particles from pp collisions.

Fig. 27. $\mathrm{Ed}^{3} \sigma / \mathrm{dp}^{3}$ versus $\sqrt{\mathrm{s}}$ for $\mathrm{pp} \rightarrow \mathrm{pX}$ and $\mathrm{pp} \rightarrow \overline{\mathrm{pX}}$. Solid lines are drawn through data points with same $\left(x, p_{T}\right)$; dashed lines are interpolations from data to indicate location of same $\left(x_{R}, p_{T}\right)$.

Fig. 28. $\mathrm{E} \mathrm{d}^{3} \sigma / \mathrm{dp}^{3}$ versus $\sqrt{\mathrm{s}}$ for $\mathrm{pp} \rightarrow \mathrm{K}^{+} \mathrm{X}$ and $\mathrm{pp} \rightarrow \mathrm{K}^{-} \mathrm{X}$. Solid lines join data points with same $\left(x, p_{T}\right)$; dashed lines are interpolations from data to indicate location of same $\left(x_{R}, p_{T}\right)$.

Fig. 29. $E d^{3} \sigma / d^{3}$ versus $\sqrt{s}$ for $p p \rightarrow \pi^{\circ} X$ and $p p \rightarrow \pi^{-} X$. Solid lines join data points with same $\left(x, p_{T}\right)$; dashed lines are interpolations from data to indicate location of same $\left(x_{R}, p_{T}\right)$.

Fig. 30. Speculation on the single-particle radial distribution for charged particles in (a) $\pi^{-} p_{3}$ (b) $\mathrm{K}^{+} \mathrm{p}$, (c) $\overline{\mathrm{PP}}$ and (d) $\mathrm{e}^{+} \mathrm{e}$ inter actions. Scales in $E d^{3} \sigma / \mathrm{dp}^{3}$ are arbitrary. 